\title{
Experimental Study and Direct Strength Method for Cold-Formed Steel Built-Up I-Sectional Columns under Axial Compression
}

\author{
Xingyou Yao (iD) ${ }^{1,2}$ \\ ${ }^{1}$ Jiangxi Province Key Laboratory of Hydraulic and Civil Engineering Infrastructure Security, Nanchang Institute of Technology, \\ Nanchang 330099, China \\ ${ }^{2}$ School of Civil Engineering and Architecture, Nanchang Institute of Technology, Nanchang 330099, China \\ Correspondence should be addressed to Xingyou Yao; yaoxingyoujd@163.com
}

Received 21 February 2021; Revised 8 June 2021; Accepted 13 July 2021; Published 22 July 2021

Academic Editor: Jose Renato de Sousa

Copyright (c) 2021 Xingyou Yao. This is an open access article distributed under the Creative Commons Attribution License, which permits unrestricted use, distribution, and reproduction in any medium, provided the original work is properly cited.

\begin{abstract}
The objective of this paper is to investigate the buckling behavior and design method of the ultimate strength for the coldformed steel (CFS) built-up I-sectional columns under axial compression which failed in distortional buckling and interactive buckling. A total of 56 CFS built-up I-sectional columns subjected to axial compression were tested, and the different buckling modes and ultimate strengths were analyzed in detail by varying the thickness, the length, the spacing of screws, the end fastener group, and the cross-sectional dimensions of CFS built-up I-sectional columns. It was shown in the test that noticeable interaction of local and distortional buckling or interaction of local, distortional, and global buckling was observed for the built-up I-sectional columns with different lengths and cross-sectional dimensions. A finite element model (FEM) was developed and validated with experimental results. A further parametric study has been conducted including different cross sections and slenderness ratios for the built-up I-sectional columns. The load-carrying capacities obtained from the experimental and numerical study were used to investigate the feasibility of the current direct strength method (DSM) when DSM was applied to CFS built-up I-sectional columns. The comparison results showed that the current DSM is not safe for CFS builtup columns failed in distortional buckling and interactive buckling. Therefore, the improved design formulas were proposed, and their accuracy was verified by using finite element analysis (FEA) and experimental results of CFS built-up I-sectional columns subjected to axial compression.
\end{abstract}

\section{Introduction}

The use of CFS members has become increasingly popular during the past few decades because CFS has high strength and stiffness, convenient manufacturing and transportation, and a high construction speed. In many countries, CFS can be found as a common structural style to construct the commercial and residential buildings. Recently, the multistorey building and the long-span frame constructed by using CFS members have appeared in many fields. These structures require higher axial or bending capacity, so multiple members are connected together by screws or welding to form a built-up CFS member as a common solution in current practice when the individual section is not able to carry the required load.
Currently, the effective width method (EWM) $[1,2]$ and the DSM [2] are two basic design methods for CFS members in CFS design specifications. However, the EWM used to design CFS built-up I-sectional members in some codes is highly simplified. "Technical Specification for Low-Rise Cold-Formed Thin-Walled Steel Buildings" (JGJ227-2011) [1] specifies that the ultimate strength is the summation of the ultimate strength of both individual member for the built-up I-section column. The 2016 AISI Specification [2] specifies that the ultimate strength of built-up I-sectional columns should be predicted with a modified slenderness ratio $(\mathrm{KL} / r)_{m}$ by considering the effect of shear force between individual sections; the modified slenderness ratio can be predicted by using the following formula: 


$$
\left(\frac{\mathrm{KL}}{r}\right)_{m}=\sqrt{\left(\frac{\mathrm{KL}}{r}\right)_{0}^{2}+\left(\frac{a}{r_{i}}\right)^{2}}
$$

where $(\mathrm{KL} / r)_{0}$ is the entire slenderness ratio of the built-up section, $a$ is the spacing of intermediate fasteners, and $r_{i}$ is the minimum radius of gyration of gross cross-sectional area of an individual section in a built-up column. It is needed to note that this method is mainly focus on the overall buckling by increasing the slenderness ratio to decrease the load capacity of the CFS built-up column. However, the CFS built-up columns are often governed by distortional buckling, local buckling, and interaction with global buckling. The modified slenderness method cannot consider the effect of the fastener on distortional buckling, local buckling, and interaction buckling.

Some works have been conducted by researchers to study the buckling modes and ultimate strength of axially-compressed built-up columns. The experiment of built-up I-sectional columns formed with two channels and connected with screws conducted by Stones [3] showed that predicted ultimate strength of built-up columns subjected to axial compression by using AISI design provision is conservative for columns with thin section and nonconservative for columns with thick section. Whittle's study reported the same results [4]. The tests on $60 \mathrm{CFS}$ built-up columns under axial compression were performed by Roy et al. [5]. The study results indicated that AISI standard is unconservative for short columns failed by local buckling and overconservative for the intermediate and slender columns failed by overall buckling.

The interaction buckling behavior and DSM for the built-up columns have been studied by other researchers. The interaction local bucking and global buckling were investigated for the CFS lipped channel built-up columns by David et al. [6]. Rational design approaches about DSM were proposed. The composite action about the CFS built-up stud and the sheathing stud was studied by David et al. [7]. The research results indicated that the different combinations of sheathing and the end boundary conditions had great impact on the strength and stability of the stud. The analytical formulas to predict the distortional buckling [8] and local buckling [9] for built-up members were also presented, which consider the effects of the length of columns and the end condition. The effects of cross section and length of the member on the buckling behavior of the built-up columns connected with four U-sections were tested by Anbarasu et al. [10], which showed that DSM cannot predict the ultimate strength of this kind of section. Anbarasu [11] numerically investigated the structural behavior and presented the design method of the CFS built-up column formed with lipped sigma sections based on DSM.

A series of buckling tests and numerical analyses were performed by some researchers [12-18] including various types of CFS built-up cross-sections. The axially-compressed and eccentrically-compressed built-up box columns were studied by experiment and finite element analysis [12] considering the slenderness ratio and ratio of width to thickness of the section. Li et al. $[13,14]$ investigated the ultimate strength of CFS box columns by test and numerical analysis and proposed the design method to predict the load capacities of the built-up box members. Georgieva et al. [15-18] studied a series of CFS built-up columns formed with zee, sigma, and track sections. The comparison on ultimate strength between test results and results calculated by using presented DSM which considers the buckling interaction was completed.

Other research studies on CFS multilimbs built-up columns had some noteworthy innovations. Nine multilimbs CFS built-up stubs with three sections were conducted and analyzed using the finite element method by Liao et al. [19]. All specimens failed in local buckling and distortional buckling. 18 specimens of CFS three-limb built-up section columns under axial compression were tested by Liu and Zhou [20]. The calculated results showed that AISI DSM was conservative for the long column and middle length column, while nonconservative for the short column. The numerical research about CFS quadruple-C built-up section members was reported by Nie et al. [21]. All specimens failed in interaction of local buckling and distortional buckling. The results predicted by using EWM and DSM were lower slightly than test and FEA results when the slenderness ratio of the column was less than 50, while the results calculated were too conservative when the slenderness ratio of the column was more than 50 . The numerical parametric study on the structural behavior of battened cold-formed steel built-up box columns composed of four lipped angles was reported by considering the sectional compactness of the lipped angle section, batten spacing, and global column slenderness [22]. The improved design procedure was proposed in the basic of AS/NZS specifications and European standards. The numerical investigation was conducted for the axial capacity and the nonlinear deformation response of pin-ended CFS built-up columns connected with spacers by varying the plate slenderness of the lipped channels, unbraced chord slenderness, and global slenderness [23]. The new design equations for the reliable design strength predictions of the CFS built-up column composed of lipped channels with spacers were proposed. The twenty built-up cold-formed steel stub columns with four different cross-sectional geometries were investigated [24]. The experiments showed a significant interaction between the individual components of the columns, and the ultimate cross-sectional capacity was much less dependent on the connector spacing within the considered range of spacing. Thirty built-up double-box columns consisting of four coldformed steel channels were experimentally tested under concentric and eccentric axial compression [25]. The methods in China and AISI S100-16 were found to be conservative for calculating the eccentric axial compression strengths of these built-up double-box columns. The closed form solution of buckling load for the built-up sections was provided by Rasmussen et al. [26].

Despite there are a large amount of research works about built-up members under axial compression, limited research studies have been reported to built-up CFS axial members about distortional buckling and interaction buckling, 
especially designing with the direct strength method. In order to analyze the distortional buckling and interactive with local and/or global buckling, the built-up CFS columns with steel grade LG550 were selected to test because these members had the large width-to-thickness ratio and were easy to cause distortional buckling and interactive buckling. This paper presented a detailed experimental results consisting of 56 CFS built-up I-sectional columns under axial compression with four different cross-sectional geometries. Then, the proven finite element model was used to study the effect of the slenderness ratio, spacing of screws, and end fastener group on axial behavior of CFS built-up I-sectional columns. Finally, the modified DSM was presented to determine the ultimate strength of CFS built-up I-sectional columns considering distortional buckling and interaction buckling.

\section{Design of Experiments}

2.1. Specimen Geometry. The 56 built-up I-sectional columns were manufactured using zinc-coated structural steel sheets LQ550 with the nominal thickness of $0.8 \mathrm{~mm}$ and $1.0 \mathrm{~mm}$. The definition of cross section is depicted in Figure 1, in which $h, b, a$, and $t$ represent the nominal height of the web, the nominal width of the flange, the nominal depth of the lip, and nominal thickness of cross section. There were four kinds of cross sections selected to test, and the nominal section dimensions are listed in Table 1 . The nominal lengths were 500,1500 , and $2000 \mathrm{~mm}$ for each kind of cross section. There were 2 specimens for the built-up columns with length $500 \mathrm{~mm}$, and the spacing of screws was $150 \mathrm{~mm}$. There were 6 specimens for the built-up columns with length $1500 \mathrm{~mm}$ and $2000 \mathrm{~mm}$, including $150 \mathrm{~mm}$ and $300 \mathrm{~mm}$ spacing of screws which satisfied the provision in the Chinese code [1], with end fastener group (EFG) and without EFG. The local $(L)$, distortional $(D)$, and global $(G)$ elastic buckling stress $\left(f_{\text {crl }}, f_{\text {crd }}\right.$, and $\left.f_{\text {cre }}\right)$ for the channel sections are listed in Table 1 . The slenderness ratio $\left(\lambda_{l}, \lambda_{d}\right.$, and $\left.\lambda_{c}\right)$ for the local, distortional, and global buckling used in the DSM and slenderness ratio $(\lambda=L / r)$ are also given in Table 1 . The screw connections are detailed in Figure 2 for the built-up I-sectional columns with the spacing of screws in $150 \mathrm{~mm}$. The specimens were labeled as depicted in Figure 3. For example, the label "DC7510-15-S150-Y-1" defines the specimen as follows: DC indicates that the section is a builtup I-section connected with double C-sections, 7510 means the nominal height of web and the nominal thickness of the section are $75 \mathrm{~mm}$ and $1.0 \mathrm{~mm}, 15$ indicates that the nominal length of the specimen is $1500 \mathrm{~mm}$, S150 represents that the spacing of screws is $150 \mathrm{~mm}$, Y means installing EFG, and 1 represents the sequence number of the same specimens. The measured dimensions of all specimens are tabulated in Table 2. The measured steel thickness is $0.794 \mathrm{~mm}$ and $0.995 \mathrm{~mm}$ for the steel with nominal thickness $0.8 \mathrm{~mm}$ and $1.0 \mathrm{~mm}$, respectively.

2.2. Material Properties. A series of coupon tests were conducted to determine the material properties of the

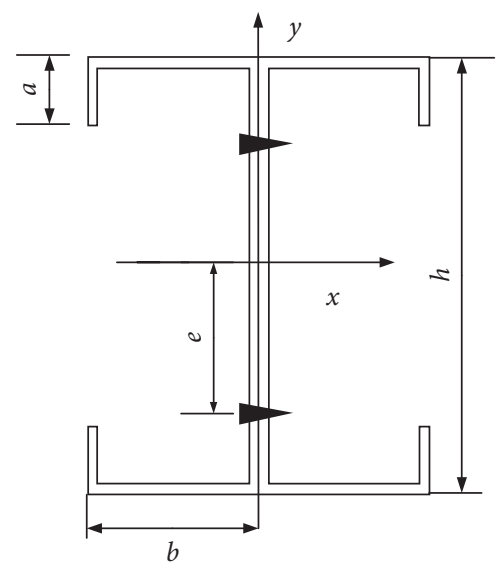

Figure 1: Built-up I-section column.

built-up columns. The coupons of grade LQ550 were cut along the longitudinal direction of specimens. For each thickness of steel sheets, 3 tensile coupons were conducted based on Chinese specifications GB/T228.1-2010 [27]. The stress-strain curves of the coupons for each thickness of steel plates are shown in Figure 4. The measured average material properties including the $0.2 \%$ proof stress $\left(f_{0.2}\right)$, initial Young's modulus $(E)$, ultimate strength $\left(f_{\mathrm{u}}\right)$, and ultimate strain $\left(\varepsilon_{\mathrm{u}}\right)$ are summarized in Table 3.

2.3. Imperfection Measurements. The initial geometric imperfection generated in forming and transporting has a great effect on the buckling behavior of the CFS column. The initial geometric imperfections along the longitudinal direction of all simple $\mathrm{C}$-sectional specimens were measured before tests and measured with an interval of $50 \mathrm{~mm}$ and $150 \mathrm{~mm}$ for specimens with length $500 \mathrm{~mm}$ and specimens with length $1500 \mathrm{~mm}$ and $2000 \mathrm{~mm}$, respectively. The measuring locations at cross section are shown in Figure 5. The locations 1, 2 (6), 3 (5), and 4 (7) indicate the deviations from flats for the initial local imperfection, the initial distortional imperfection, the initial global imperfection about the weak axis, and the initial global imperfections about the great axis, respectively. The initial imperfections along the longitudinal direction for section DC7510 series and section DC9008 series are depicted in Figure 6. The initial imperfections of the other specimens had almost the similar distribution to these specimens. The initial geometric imperfections measured indicate that the imperfections have no obvious rules along the longitudinal direction. However, it is needed to note that the initial distortional imperfections are larger than the initial local and global imperfections. Table 2 lists the maximum initial geometric imperfection magnitudes $\left(\Delta_{\max }\right)$ for all specimens.

2.4. Test Setup. The specimens were loaded by a $50 \mathrm{kN}$ hydraulic jack and reaction frame system, as shown in Figure 7. A $250 \mathrm{~mm} \times 200 \mathrm{~mm} \times 8 \mathrm{~mm}$ thick steel platen was rigidly fixed to the upper actuator, and another $250 \mathrm{~mm} \times 200 \mathrm{~mm} \times 8 \mathrm{~mm}$ thick steel platen was put on the lower steel block. These two steel platens were parallel by 
TABLE 1: Nominal dimensions of sections.

\begin{tabular}{|c|c|c|c|c|c|c|c|c|c|c|c|c|}
\hline Specimen & $h(\mathrm{~mm})$ & $b(\mathrm{~mm})$ & $a(\mathrm{~mm})$ & $t(\mathrm{~mm})$ & $L(\mathrm{~mm})$ & $f_{\mathrm{crl}}$ & $f_{\text {crd }}$ & $f_{\text {cre }}$ & $\lambda_{l}$ & $\lambda_{d}$ & $\lambda_{c}$ & $\lambda$ \\
\hline DC7510 & 75 & 40 & 10 & 1 & $\begin{array}{c}500 \\
1500 \\
2000 \\
\end{array}$ & 186 & 291.4 & $\begin{array}{c}783.6 \\
122.14 \\
73.16 \\
\end{array}$ & $\begin{array}{l}2.05 \\
0.81 \\
0.63 \\
\end{array}$ & 1.37 & $\begin{array}{l}0.84 \\
2.12 \\
2.74 \\
\end{array}$ & $\begin{array}{c}33.31 \\
99.94 \\
133.25 \\
\end{array}$ \\
\hline DC10008 & 100 & 90 & 10 & 0.8 & $\begin{array}{c}500 \\
1500 \\
2000 \\
\end{array}$ & 54 & 60 & $\begin{array}{c}1562 \\
130.2 \\
104.4 \\
\end{array}$ & $\begin{array}{l}5.38 \\
1.55 \\
1.39 \\
\end{array}$ & 3.03 & $\begin{array}{l}0.59 \\
2.06 \\
2.30 \\
\end{array}$ & $\begin{array}{l}15.45 \\
46.35 \\
61.79 \\
\end{array}$ \\
\hline DC9008 & 90 & 50 & 15 & 0.8 & $\begin{array}{c}500 \\
1500 \\
2000 \\
\end{array}$ & 84 & 204 & $\begin{array}{c}1131.1 \\
177.6 \\
103.8 \\
\end{array}$ & $\begin{array}{l}3.67 \\
1.45 \\
1.11 \\
\end{array}$ & 1.64 & $\begin{array}{l}0.70 \\
1.76 \\
2.30 \\
\end{array}$ & $\begin{array}{c}25.78 \\
77.33 \\
103.10 \\
\end{array}$ \\
\hline DC8008 & 80 & 60 & 10 & 0.8 & $\begin{array}{c}500 \\
1500 \\
2000\end{array}$ & 94.8 & 120.7 & $\begin{array}{c}952 \\
123.8 \\
73.9\end{array}$ & $\begin{array}{l}3.17 \\
1.14 \\
0.88\end{array}$ & 2.13 & $\begin{array}{l}0.76 \\
2.11 \\
2.73\end{array}$ & $\begin{array}{l}22.56 \\
67.67 \\
90.23\end{array}$ \\
\hline
\end{tabular}

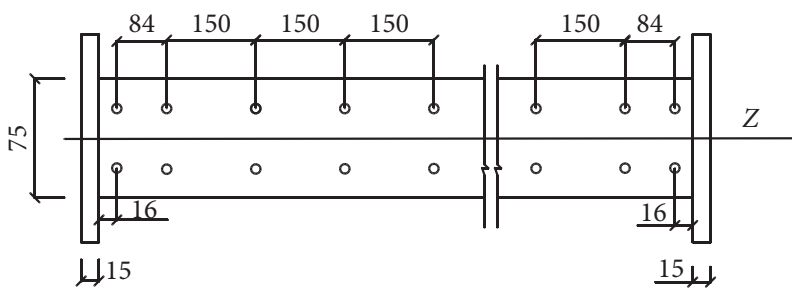

(a)

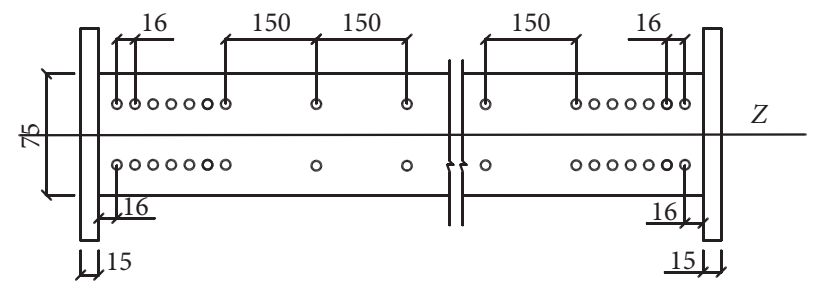

(b)

Figure 2: Screw arrangement of built-up I-sectional columns. (a) Built-up I-sectional columns without the end fastener group. (b) Built-up I-sectional columns with the end fastener group.

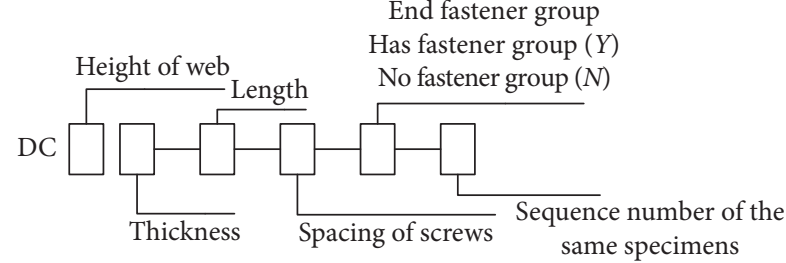

FIGURE 3: Specimen labeling.

adjusting the lower steel block. The column specimens are put directly on the steel platen as they were compressed. Friction between the column ends and the steel platens was the only lateral forces that restrained the column cross section under load. The axial compressive loading was obtained from the load cell positioned at the top of the test specimens. A total of 7 displacement transducers (D1 to D7) were installed to measure the displacement of specimens at specified points, as illustrated in Figure 8. The lateral displacement transducers D1-D6 were employed at the midheight of specimens, and the axial displacement transducer D7 was put at the upper loaded plate to obtain the axial shorten displacements of the specimens. All the test data could be obtained by using the YJ16 data acquisition instrument.

\section{Experiment Results}

3.1. Buckling and Failure Mode. Table 4 presents the test results of 56 built-up I-sectional columns under axial compression, including the buckling modes by visual inspection and load capacities, where $L, D$, and $G$ represent local buckling, distortional buckling, and overall buckling, respectively. Two types of buckling modes were found in the tests, as shown in Table 4. The section DC7510-20 series and the section DC8008-20 series failed in interaction of local, distortional, and overall buckling modes. The interaction of local and distortional buckling was the main buckling modes for the other specimens.

3.1.1. Built-Up Columns with the Nominal Length of $500 \mathrm{~mm}$. The typical buckling modes of specimens with the nominal length of $500 \mathrm{~mm}$ are illustrated in Figure 9. All specimens failed in interaction of distortional buckling and local buckling and displayed a distortional buckling half-wave and many local buckling half-waves. There was no evident deformation during the initial stage of loading, and distortional buckling (Figure 9(a)) was observed with increasing of loading for section DC7510 series, section DC10008 series, and section DC8008 series. Then, local buckling in web (Figure 9(a)) was observed for all specimens with increasing of loading. Meanwhile, local buckling in the flange (Figure 9(c)) occurred for section DC10008 series and DC8008 series because the flanges of these sections have large ratio of width to thickness. The specimens finally failed because of the plastic crush which resulted from the large buckling deformation (Figure 9(d)). For section DC9008 series, local buckling in web (Figure 9(b)) appeared before the observation of distortional buckling and failed with plastic crush (Figure 9(d)) as same as other specimens. There 
TABle 2: Measured dimensions of specimens.

\begin{tabular}{|c|c|c|c|c|c|c|}
\hline Specimen & Section & $L_{1}(\mathrm{~mm})$ & $h_{1}(\mathrm{~mm})$ & $b_{1}(\mathrm{~mm})$ & $a_{1}(\mathrm{~mm})$ & $\Delta_{\max }(\mathrm{mm})$ \\
\hline \multirow{2}{*}{ DC7510-05-S150-N-1 } & $\mathrm{a}$ & \multirow{2}{*}{499.5} & 73.88 & 39.46 & 9.14 & \multirow{2}{*}{1.62} \\
\hline & $\mathrm{b}$ & & 73.7 & 39.4 & 9.84 & \\
\hline \multirow{2}{*}{ DC7510-05-S150-N-2 } & $\mathrm{a}$ & \multirow{2}{*}{499.5} & 73.72 & 39.28 & 9.23 & \multirow{2}{*}{1.42} \\
\hline & $\mathrm{b}$ & & 73.21 & 39.35 & 9.08 & \\
\hline \multirow{2}{*}{ DC7510-15-S150-N-1 } & $\mathrm{a}$ & \multirow{2}{*}{1500} & 74.2 & 39.21 & 9.64 & \multirow{2}{*}{1.22} \\
\hline & $\mathrm{b}$ & & 73.89 & 39.07 & 9.25 & \\
\hline \multirow{2}{*}{ DC7508-15-S150-N-2 } & $\mathrm{a}$ & \multirow{2}{*}{1500} & 73.59 & 39.85 & 9.19 & \multirow{2}{*}{0.89} \\
\hline & $\mathrm{b}$ & & 73.94 & 39.52 & 9.78 & \\
\hline \multirow{2}{*}{ DC7508-15-S300-N-1 } & $\mathrm{a}$ & \multirow{2}{*}{1500} & 73.99 & 39 & 9.01 & \multirow{2}{*}{1.07} \\
\hline & $\mathrm{b}$ & & 73.06 & 39.89 & 9.78 & \\
\hline DC7510-15-S300-N-2 & $\mathrm{a}$ & 1500 & 74.16 & 39.96 & 9.94 & 122 \\
\hline $0 \mathrm{C} / 510-15-3300-\mathrm{N}-2$ & $\mathrm{~b}$ & 1500 & 75.58 & 39.05 & 10.69 & 1.22 \\
\hline DC7510-15-S150-Y-1 & $\mathrm{a}$ & 1500 & 75 & 39.65 & 10.08 & 053 \\
\hline DC/510-15-S150-Y-1 & $\mathrm{b}$ & 1500 & 75 & 39.77 & 9.9 & 0.53 \\
\hline DC7510-15-S300-Y-1 & $\mathrm{a}$ & 1500 & 73.77 & 39.65 & 10.08 & 078 \\
\hline $10 / 210-15-3500-1-1$ & $\mathrm{~b}$ & 1500 & 74.27 & 39.77 & 9.9 & 0.78 \\
\hline DC7510-20-S150-N-1 & $\mathrm{a}$ & 199985 & 75.75 & 39.86 & 9.89 & 082 \\
\hline $0 \mathrm{C} / 510-20-5150-\mathrm{N}-1$ & $\mathrm{~b}$ & 1999.85 & 75.47 & 39.76 & 9.04 & 0.82 \\
\hline DC7510-20-S150-N-2 & $\mathrm{a}$ & & 75.21 & 39.97 & 9.32 & \\
\hline DC/510-20-S150-N-2 & $\mathrm{b}$ & 1999.8 & 74.8 & 39.82 & 9.31 & 0.56 \\
\hline DC7510-20-S300-N-1 & $\mathrm{a}$ & 109065 & 74.15 & 40.13 & 9.99 & 075 \\
\hline $0 \mathrm{C} / 510-20-5300-\mathrm{N}-1$ & $\mathrm{~b}$ & 1999.65 & 75.31 & 39.79 & 10.08 & 0.75 \\
\hline DC7510-20-S300-N-2 & $\mathrm{a}$ & & 75.96 & 39.82 & 9.58 & \\
\hline$D C / 510-20-5300-N-2$ & $\mathrm{~b}$ & 1999.95 & 75.31 & 39.81 & 9.93 & 1.04 \\
\hline DC7510-20-S150-Y-1 & $\mathrm{a}$ & 199955 & 75.56 & 39.78 & 9.74 & 122 \\
\hline $0 \mathrm{C} / 510-20-5150-Y-1$ & $\mathrm{~b}$ & 1999.55 & 74.48 & 40.68 & 9.95 & 1.22 \\
\hline DC7510-20-S300-Y-1 & $\mathrm{a}$ & 19998 & 74.58 & 39.93 & 9.91 & \\
\hline & $\mathrm{b}$ & 1999.8 & 75.76 & 39.64 & 9.67 & 1.28 \\
\hline DC10008-05-S150-N-1 & $\mathrm{a}$ & 4995 & 97.91 & 89.17 & 9.91 & 053 \\
\hline DC10008-05-S150-N-I & $\mathrm{b}$ & 499.5 & 97.48 & 89.91 & 9.44 & 0.53 \\
\hline DC10008-05-S150-N-2 & $\mathrm{a}$ & 4995 & 98.45 & 89.47 & 9.45 & 062 \\
\hline DCI0008-05-S150-N-2 & $\mathrm{b}$ & 499.5 & 97.99 & 88.79 & 9.7 & 0.62 \\
\hline DC10008-15-S150-N-1 & $\mathrm{a}$ & 140925 & 99.13 & 89.99 & 9.29 & 103 \\
\hline DCI0008-15-3150-N-1 & $\mathrm{b}$ & 1499.25 & 99.21 & 89.41 & 9.72 & 1.03 \\
\hline & $\mathrm{a}$ & & 98.96 & 89.74 & 9.82 & \\
\hline DC10008-15-S150-N-2 & $\mathrm{b}$ & 1499.25 & 98.97 & 89.54 & 9.4 & 1.15 \\
\hline $\mathrm{DC} 10008-15-\mathrm{S} 300-\mathrm{N} 1$ & $\mathrm{a}$ & 14901 & 97.89 & 89.9 & 9.59 & 128 \\
\hline DC10008-15-3500-N-1 & $\mathrm{b}$ & 1499.1 & 97.54 & 88.72 & 9.46 & 1.28 \\
\hline & $\mathrm{a}$ & & 95.58 & 89.82 & 9.18 & \\
\hline DC10008-15-S300-N-2 & $\mathrm{b}$ & 1499.25 & 98.2 & 89.7 & 8.99 & 1.08 \\
\hline DC10008-15-S150-Y-1 & $\mathrm{a}$ & 1500 & 98.55 & 89.78 & 9.77 & 085 \\
\hline & $\mathrm{b}$ & & 99.4 & 89.54 & 9.73 & \\
\hline & $\mathrm{a}$ & & 98.98 & 90.38 & 8.9 & \\
\hline DC10008-15-S300-Y-1 & $\mathrm{b}$ & 1500 & 99.27 & 89.41 & 9.79 & 0.96 \\
\hline DC10008-20-S150-N-1 & $\mathrm{a}$ & 2000 & 98.03 & 89.56 & 10.04 & 120 \\
\hline & $\mathrm{b}$ & & 99.66 & 89.2 & 9.65 & \\
\hline DC10008-20-S150-N-2 & $\mathrm{a}$ & 199995 & 99.53 & 89.6 & 9.52 & 118 \\
\hline DC10008-20-S150-N-2 & $\mathrm{b}$ & 1999.95 & 99.22 & 89.42 & 9.73 & 1.18 \\
\hline DC10008-20-S300-N-1 & $\mathrm{a}$ & & 99.14 & 89.49 & 9.63 & 110 \\
\hline & $\mathrm{b}$ & 2000.05 & 98.88 & 89.75 & 9.62 & 1.10 \\
\hline DC10008-20-S300-N-2 & $\mathrm{a}$ & 199995 & 99.57 & 89.62 & 9.3 & \\
\hline DC10008-20-S300-N-2 & $\mathrm{b}$ & 1999.95 & 100.8 & 89.06 & 9.98 & 0.96 \\
\hline DC10008-20-S150-Y-1 & $\mathrm{a}$ & 2000 & 100.0 & 89.09 & 9.9 & 088 \\
\hline & $\mathrm{b}$ & 2000 & 99.61 & 89.08 & 9.64 & 0.88 \\
\hline DC10008-20-S300-Y-1 & $\mathrm{a}$ & 2000 & 99.48 & 89.32 & 9.36 & 084 \\
\hline DC10008-20-5300-Y-1 & $\mathrm{b}$ & 2000 & 99.43 & 89.25 & 9.38 & 0.84 \\
\hline DC9008-05-S150-N-1 & $\mathrm{a}$ & 49915 & 88.75 & 49.41 & 14.55 & 0.59 \\
\hline & $\mathrm{b}$ & 499.15 & 88.26 & 49.16 & 14.76 & 0.59 \\
\hline DC9008-05-S150-N-2 & $\mathrm{a}$ & 4991 & 89.26 & 49.03 & 15.83 & 121 \\
\hline DC9008-05-5150-N-2 & $\mathrm{b}$ & 499.1 & 88.51 & 49.19 & 15.11 & 1.21 \\
\hline
\end{tabular}


TABle 2: Continued.

\begin{tabular}{|c|c|c|c|c|c|c|}
\hline Specimen & Section & $L_{1}(\mathrm{~mm})$ & $h_{1}(\mathrm{~mm})$ & $b_{1}(\mathrm{~mm})$ & $a_{1}(\mathrm{~mm})$ & $\Delta_{\max }(\mathrm{mm})$ \\
\hline \multirow{2}{*}{ DC9008-15-S150-N-1 } & $\mathrm{a}$ & \multirow{2}{*}{1499.5} & 89.4 & 49.57 & 14.68 & \multirow{2}{*}{0.65} \\
\hline & $\mathrm{b}$ & & 89.3 & 49.52 & 14.47 & \\
\hline \multirow{2}{*}{ DC9008-15-S150-N-2 } & $\mathrm{a}$ & \multirow{2}{*}{1499.35} & 89.14 & 49.51 & 14.51 & \multirow{2}{*}{1.20} \\
\hline & $\mathrm{b}$ & & 89.37 & 49.89 & 14.13 & \\
\hline \multirow{2}{*}{ DC9008-15-S300-N-1 } & $\mathrm{a}$ & \multirow{2}{*}{1499.3} & 87.55 & 49.44 & 14.88 & \multirow{2}{*}{0.47} \\
\hline & $\mathrm{b}$ & & 89.3 & 49.75 & 14.49 & \\
\hline \multirow{2}{*}{ DC9008-15-S300-N-2 } & $\mathrm{a}$ & \multirow{2}{*}{1500} & 90.1 & 50.25 & 14.9 & \multirow{2}{*}{0.68} \\
\hline & $\mathrm{b}$ & & 89.9 & 49.8 & 15.08 & \\
\hline \multirow{2}{*}{ DC9008-15-S150-Y-1 } & $\mathrm{a}$ & \multirow{2}{*}{1499.5} & 89.86 & 49.33 & 14.31 & \multirow{2}{*}{1.06} \\
\hline & $\mathrm{b}$ & & 90.03 & 49.06 & 14.57 & \\
\hline \multirow{2}{*}{ DC9008-15-S300-Y-1 } & $\mathrm{a}$ & \multirow{2}{*}{1499.25} & 90.05 & 48.53 & 15.34 & \multirow{2}{*}{0.73} \\
\hline & $\mathrm{b}$ & & 89.98 & 49.82 & 14.39 & \\
\hline \multirow{2}{*}{ DC9008-20-S150-N-1 } & $\mathrm{a}$ & & 89.27 & 49.37 & 14.87 & 103 \\
\hline & $\mathrm{b}$ & 2000 & 89.76 & 49.35 & 14.77 & 1.03 \\
\hline & $\mathrm{a}$ & & 90.29 & 49.52 & 14.2 & \\
\hline DC9008-20-S150-N-2 & $\mathrm{b}$ & 1999.9 & 89.86 & 49.73 & 14.15 & 0.69 \\
\hline DC9008-20-S300-N-1 & $\mathrm{a}$ & 2000 & 90.18 & 49.33 & 14.45 & 079 \\
\hline DC9008-20-5300-N-1 & $\mathrm{b}$ & & 89.12 & 49.61 & 14.6 & \\
\hline DC9008-20-S300-N-2 & $\mathrm{a}$ & 199995 & 90.2 & 48.72 & 14.9 & 087 \\
\hline DC9008-20-5300-N-2 & $\mathrm{b}$ & 1999.95 & 89.03 & 49.48 & 14.78 & 0.87 \\
\hline DC9008-20-S150-Y-1 & $\mathrm{a}$ & 2000 & 88.67 & 49.51 & 15.25 & 103 \\
\hline DC $9008-20-5150-Y-1$ & $\mathrm{~b}$ & 2000 & 89.94 & 49.32 & 14.6 & 1.03 \\
\hline DC9008-20-S300-Y-1 & $\mathrm{a}$ & 199995 & 90.31 & 49.43 & 14.21 & 111 \\
\hline DC9008-20-5300-Y-1 & $\mathrm{b}$ & 1999.95 & 89.43 & 48.85 & 15.1 & 1.11 \\
\hline DC8008-05-S150-N-1 & $\mathrm{a}$ & 49945 & 79.05 & 59.1 & 9.83 & \\
\hline & $\mathrm{b}$ & 499.45 & 78.69 & 58.78 & 9.58 & 0.78 \\
\hline DC8008-05-S150-N-2 & $\mathrm{a}$ & 4095 & 76.55 & 59.12 & 9.98 & 062 \\
\hline DC8008-05-3150-N-2 & $\mathrm{b}$ & 499.5 & 77.99 & 59.45 & 9.9 & 0.63 \\
\hline DC8008-15-S150-N-1 & $\mathrm{a}$ & 14995 & 80.75 & 59.57 & 9.47 & 123 \\
\hline DC8008-15-S150-N-1 & $\mathrm{b}$ & 1499.5 & 79.54 & 59.63 & 9.64 & 1.23 \\
\hline DC8008-15-S150-N-2 & $\mathrm{a}$ & & 80.19 & 59.22 & 9.79 & 115 \\
\hline DC8008-15-S150-N-2 & $\mathrm{b}$ & 1499.5 & 79.56 & 59.33 & 9.84 & 1.15 \\
\hline DC8008-15-S300-N-1 & $\mathrm{a}$ & 14991 & 79.46 & 59.99 & 8.92 & 094 \\
\hline DC8008-15-S300-N-1 & $\mathrm{b}$ & 1499.1 & 79.12 & 59.65 & 9.55 & 0.94 \\
\hline DC8008-15-S300-N-2 & $\mathrm{a}$ & 1500 & 80 & 60 & 10 & 0.86 \\
\hline DC8008-15-5300-N-2 & b & 1500 & 80 & 60 & 10 & 0.86 \\
\hline $\mathrm{DC} 8000 \quad 15 \mathrm{~S} 150 \mathrm{Y} 1$ & $\mathrm{a}$ & 14095 & 78.98 & 59.51 & 9.39 & 002 \\
\hline DC8008-15-S150-Y-1 & $\mathrm{b}$ & 1499.5 & 79.97 & 59.23 & 9.28 & 0.92 \\
\hline $\mathrm{DC} 8008-15-5300-\mathrm{Y}=1$ & $\mathrm{a}$ & 14993 & 80.11 & 59.67 & 9.28 & 112 \\
\hline DC $8008-15-5300-1-1$ & $\mathrm{~b}$ & 1499.3 & 79.71 & 59.65 & 9.57 & 1.12 \\
\hline & $\mathrm{a}$ & & 79.57 & 58.49 & 10.2 & \\
\hline DC8008-20-S150-N-1 & $\mathrm{b}$ & 1999.95 & 79.41 & 58.42 & 10.27 & 0.88 \\
\hline 2 & $\mathrm{a}$ & 199085 & 79.32 & 59.12 & 10.02 & 0.91 \\
\hline DC8008-20-S150-N-2 & $\mathrm{b}$ & 1999.85 & 78.87 & 59.7 & 9.53 & 0.91 \\
\hline $\mathrm{DC} 8000_{-2} 20_{-} \mathrm{S} 300_{-}-{ }_{1}$ & $\mathrm{a}$ & & 78.53 & 59.63 & 9.86 & 0.93 \\
\hline DC8008-20-5300-N-1 & $\mathrm{b}$ & 1999.65 & 78.39 & 60.03 & 9.33 & 0.93 \\
\hline $\mathrm{DC} 8008^{-}-20_{-} \mathrm{S} 300_{-} \mathrm{N}_{-} 2$ & $\mathrm{a}$ & 2000 & 79.09 & 60.02 & 9.18 & 111 \\
\hline & b & 2000 & 79.71 & 59.51 & 9.44 & 1.11 \\
\hline DC8008-20-S150-Y 1 & $\mathrm{a}$ & & 79.86 & 59.3 & 9.93 & 108 \\
\hline DC8008-20-S150-Y-1 & $\mathrm{b}$ & 1999.95 & 80.06 & 59.33 & 9.48 & 1.08 \\
\hline DC8008-20-S300-Y-1 & $\mathrm{a}$ & 2000 & 80.78 & 59.16 & 9.42 & 103 \\
\hline & $\mathrm{b}$ & & 79.43 & 59.32 & 9.67 & 1.03 \\
\hline
\end{tabular}

was no failure about connection screws which indicate that two C-sections in built-up specimens can work together and develop composite action.

3.1.2. Built-Up Columns with the Nominal Length of $1500 \mathrm{~mm}$. The typical buckling modes of specimens with the nominal length of $1500 \mathrm{~mm}$ are illustrated in Figure 10. All specimens failed in interaction of distortional buckling and local buckling and displayed three distortional buckling half-waves and many local buckling half-waves. The local buckling in web (Figure 10(a)) appeared gradually with increasing of loading for section DC7510 series, section DC10010 series, and section DC8008 series. 


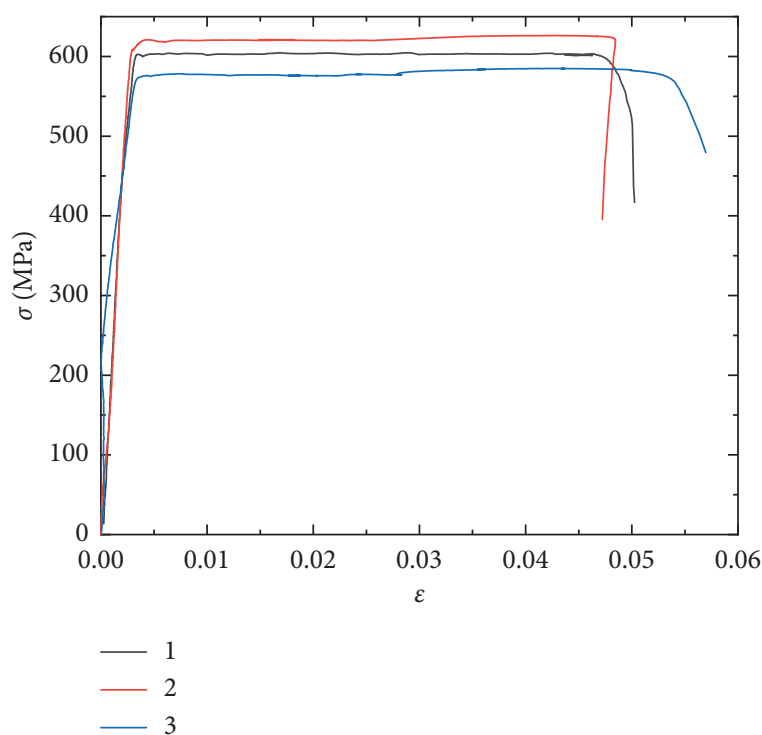

(a)

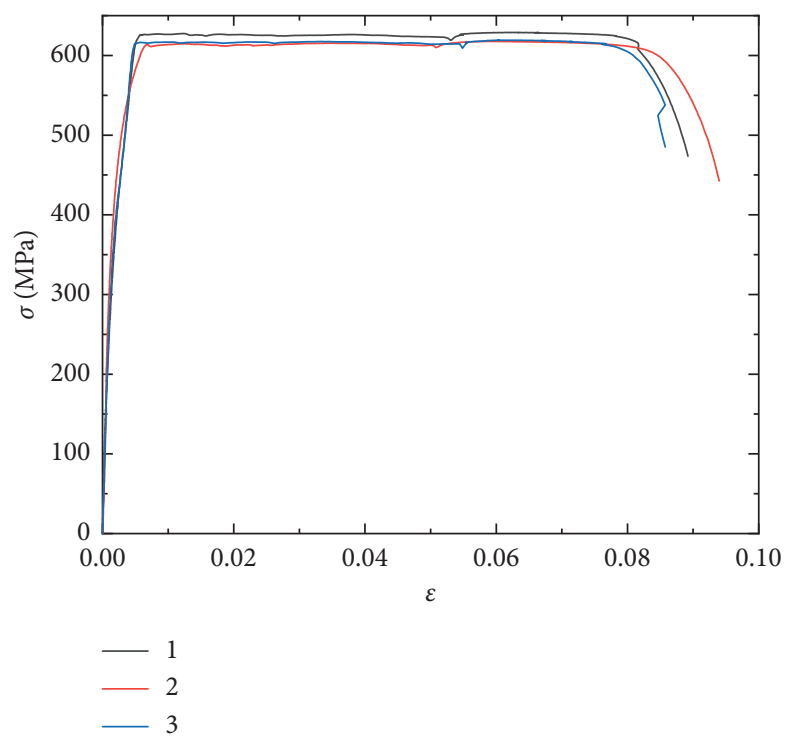

(b)

Figure 4: Stress-strain curve of coupon tests. (a) $t=0.8 \mathrm{~mm}$. (b) $t=1.00 \mathrm{~mm}$.

TABLE 3: Mean values of coupon tests.

\begin{tabular}{lcccc}
\hline$t(\mathrm{~mm})$ & $f_{0.2}(\mathrm{MPa})$ & $f_{\mathrm{u}}(\mathrm{MPa})$ & $E(\mathrm{MPa})$ & $\varepsilon_{\mathrm{u}}(\%)$ \\
\hline 0.8 & 600 & 602 & $2.16 \times 10^{5}$ & 7.19 \\
1.00 & 620 & 634 & $2.15 \times 10^{5}$ & 8.92 \\
\hline
\end{tabular}

Note. $t$ is the nominal thickness.

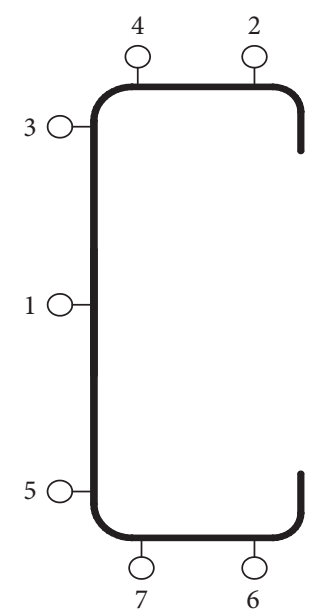

FIGURE 5: Measure location of the initial geometric imperfection.

Meanwhile, local buckling in the flange (Figure 10(b)) can be found for section DC10008 and DC8008. Then, distortional buckling (Figure 9(c)) was observed for all specimens with increasing of loading. The specimens finally collapsed with the plastic crush near its midheight (Figure 9(d)). For section DC10008 series, distortional buckling (Figure 9(c)) was observed before the local buckling, and it failed with plastic crush (Figure 9(d)) as same as other specimens. There was no failure about connection screws.
3.1.3. Built-Up Columns with the Nominal Length of $2000 \mathrm{~mm}$. The typical buckling modes of specimens with nominal length of $2000 \mathrm{~mm}$ are presented in Figure 11. For section DC7510 series and section DC8008 series (Figure 11(a)), the local buckling in web appeared with the increasing of loading. Then specimens displayed the distortional buckling with the increasing of loading. The specimens finally collapsed with the global flexural buckling because these sections had larger slenderness ratio than other sections. However, for section DC10008 series and section DC9008 series, the interaction of distortional buckling and local buckling (Figure 11(b)) was observed, and they failed with plastic crush. There was no failure about connection screws.

3.2. Load and Axial Shorten Displacement Curves. Load and axial shorten displacement curves and load and lateral displacement curves for section DC7510 series are provided in Figures 12 and 13, respectively. The other load and displacement datum can be found in [28]. As shown in Figure 12, the two repeated tests displayed the almost same curve which indicated that the obtained results in the compression test are reliable. Meanwhile, it can be found that the spacing of screws changing from 300 to $150 \mathrm{~mm}$ and arranging of the end fastener group can increase the ultimate capacities and stiffness slightly. So, it may be indicated that the spacing of screws and EFG can provide some benefit for CFS built-up I-sectional columns. The displacements of displacement transducers 2 and 6 and 1 and 5 in Figure 13 indicate that the specimens displayed obvious distortional buckling displacements.

Figure 14 shows the comparison on load vs. axial displacement curves for section DC7510 series with different lengths. It can be observed that the ultimate capacity and stiffness would decrease with the increasing of length, which 


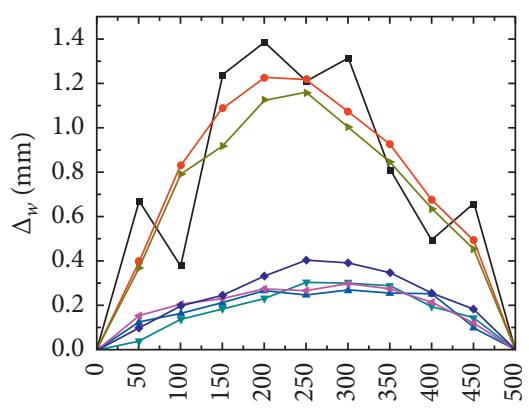

$L(\mathrm{~mm})$

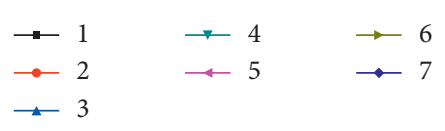

(a)

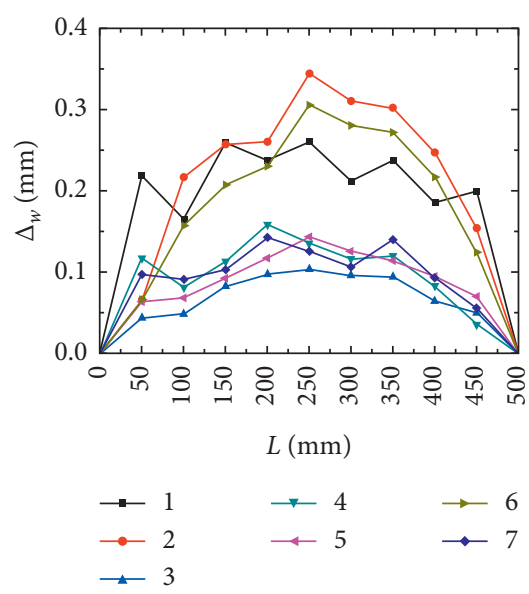

(d)

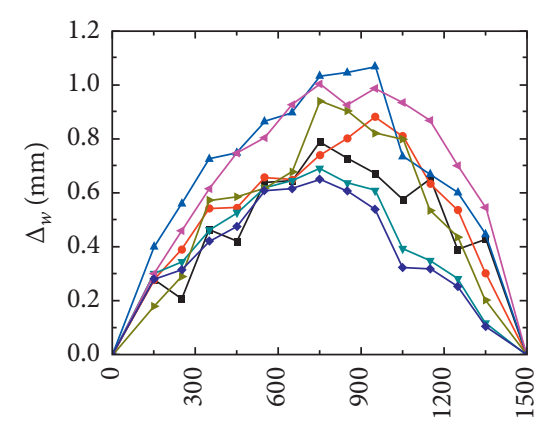

$L(\mathrm{~mm})$

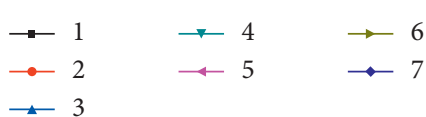

(b)

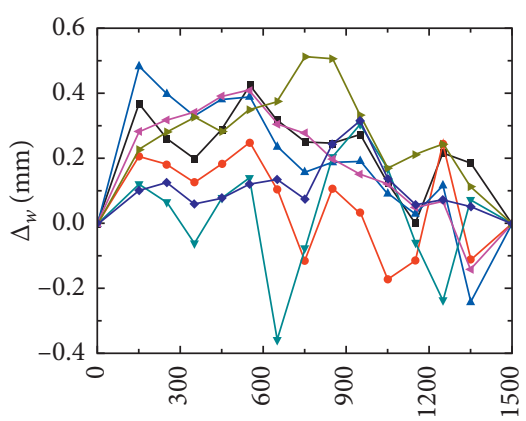

$L(\mathrm{~mm})$

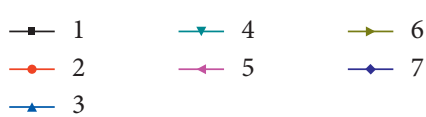

(e)
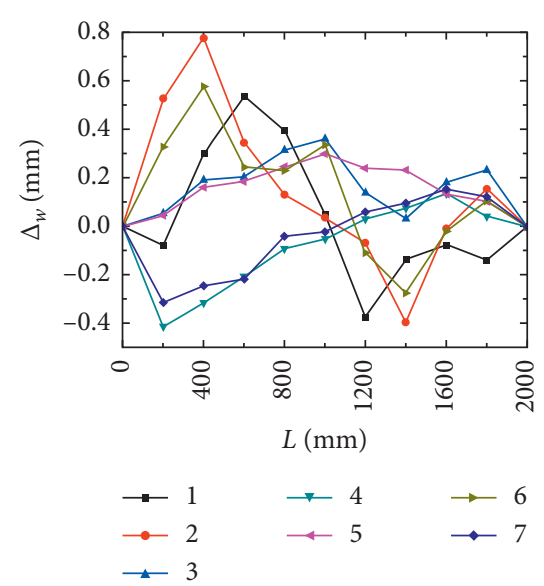

(c)
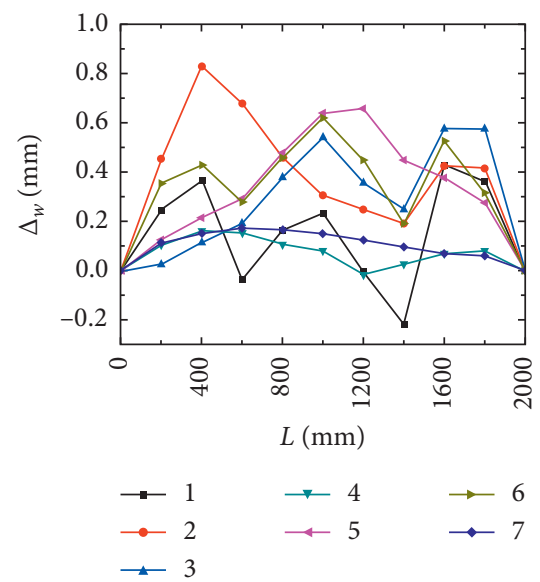

(f)

Figure 6: Initial imperfections of channel sections. (a) DC7510-05-S150-N-1. (b) DC9008-05-S150-N-1. (c) DC7510-15-S150-N-1. (d) DC9008-15-S150-N-1. (e) DC7510-20-S150-N-1. (f) DC9008-20-S150-N-1.

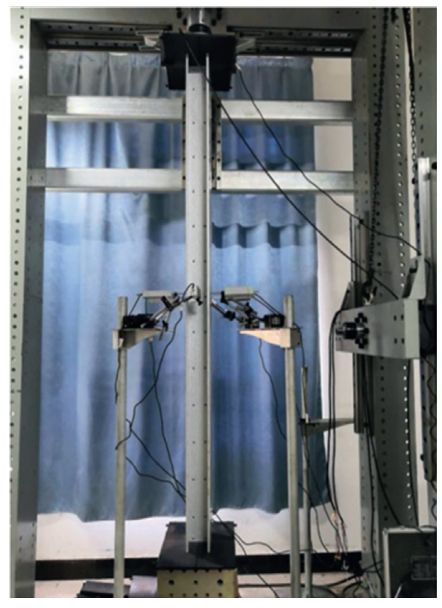

(a)

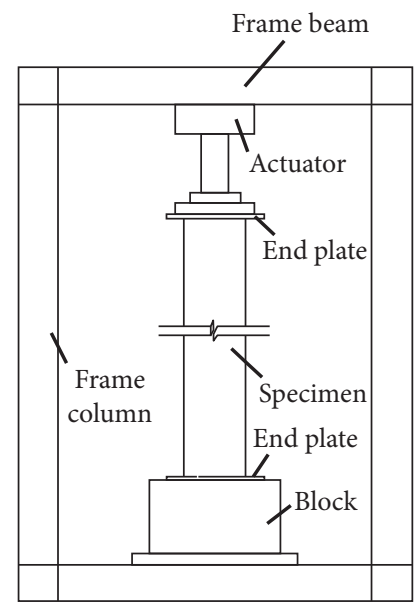

(b)

Figure 7: Test setup.

indicates that the slenderness ratio has important effect on ultimate strength and stiffness of CFS built-up I-sectional columns.
3.3. Ultimate Strength Analysis. The ultimate strengths of specimens with different sections, spacing of screws, and end fastener group are summarized in Table 4, in which $P_{\mathrm{t}}$ and 


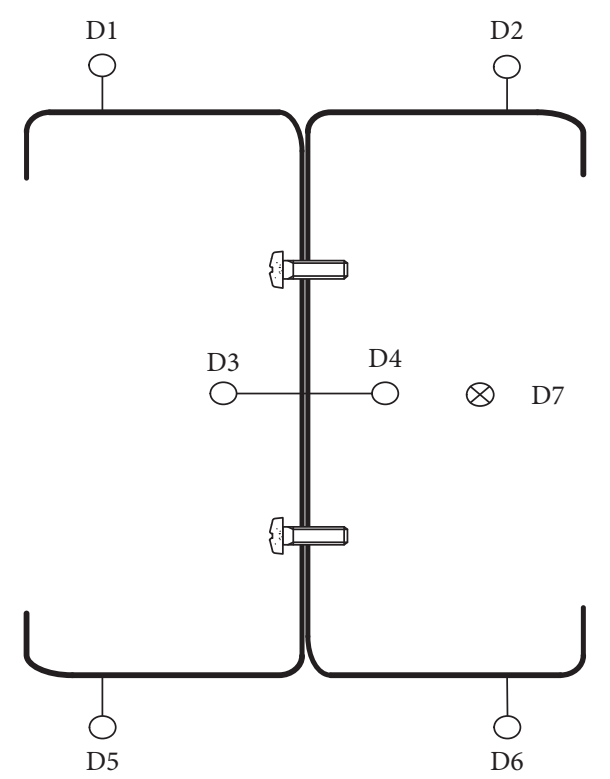

Figure 8: Arrangement of displacement transducers.

TABle 4: Comparison on buckling modes and ultimate load capacities of built-up I-sectional columns.

\begin{tabular}{|c|c|c|c|c|c|c|c|c|c|c|c|}
\hline No. & Specimen & $\begin{array}{l}\text { Test buckling } \\
\text { mode }\end{array}$ & $\begin{array}{l}\text { FEA buckling } \\
\text { mode }\end{array}$ & $P_{\mathrm{t}}(\mathrm{kN})$ & $\begin{array}{c}P_{\mathrm{ta}} \\
(\mathrm{kN})\end{array}$ & $P_{\mathrm{A}}(\mathrm{kN})$ & $\begin{array}{c}P_{\mathrm{D}} \\
(\mathrm{kN})\end{array}$ & $\begin{array}{l}\mathrm{P}_{\mathrm{MD}} \\
(\mathrm{kN})\end{array}$ & $\begin{array}{l}P_{\mathrm{t}} l \\
P_{\mathrm{A}}\end{array}$ & $\begin{array}{l}P_{\mathrm{t}} / \\
P_{\mathrm{D}}\end{array}$ & $\begin{array}{c}P_{\mathrm{t}} / \\
\mathrm{P}_{\mathrm{MD}}\end{array}$ \\
\hline 1 & $\begin{array}{c}\text { DC7510-05-S150- } \\
\text { N-1 }\end{array}$ & $\mathrm{D}+\mathrm{L}$ & $\mathrm{D}+\mathrm{L}$ & 105.44 & 108.66 & 120.92 & 126.74 & 104.29 & 0.872 & 0.832 & 1.011 \\
\hline 2 & $\begin{array}{c}\text { DC7510-05-S150- } \\
\text { N-2 }\end{array}$ & $\mathrm{D}+\mathrm{L}$ & $\mathrm{D}+\mathrm{L}$ & 111.88 & & 121 & 125.5 & 104.7 & 0.925 & 0.891 & 1.069 \\
\hline 3 & $\begin{array}{c}\text { DC7510-15-S150- } \\
\text { N-1 }\end{array}$ & $\mathrm{L}+\mathrm{D}$ & $\mathrm{L}+\mathrm{D}$ & 92.9 & 94.55 & 96.78 & 128.04 & 96.33 & 0.960 & 0.726 & 0.964 \\
\hline 4 & $\begin{array}{c}\text { DC7510-15-S150- } \\
\text { N-2 }\end{array}$ & $\mathrm{L}+\mathrm{D}$ & $\mathrm{L}+\mathrm{D}$ & 96.2 & & 96.3 & 128.5 & 95.9 & 0.999 & 0.749 & 1.003 \\
\hline 5 & $\begin{array}{c}\text { DC7510-15-S300- } \\
\text { N-1 }\end{array}$ & $\mathrm{L}+\mathrm{D}$ & $\mathrm{L}+\mathrm{D}$ & 91.8 & 92.05 & 92.92 & - & - & 0.988 & - & - \\
\hline 6 & $\begin{array}{c}\text { DC7510-15-S300- } \\
\text { N-2 }\end{array}$ & $\mathrm{L}+\mathrm{D}$ & $\mathrm{L}+\mathrm{D}$ & 92.3 & & 92.38 & - & - & 0.999 & - & - \\
\hline 7 & $\begin{array}{c}\text { DC7510-15-S150-Y- } \\
1\end{array}$ & $\mathrm{~L}+\mathrm{D}$ & $\mathrm{L}+\mathrm{D}$ & 96.5 & - & 97.66 & - & - & 0.988 & - & - \\
\hline 8 & $\begin{array}{c}\text { DC7510-15-S300-Y- } \\
2\end{array}$ & $\mathrm{~L}+\mathrm{D}$ & $\mathrm{L}+\mathrm{D}$ & 94.9 & & 100.53 & - & - & 0.944 & - & - \\
\hline 9 & $\begin{array}{c}\text { DC7510-20-S150- } \\
\text { N-1 }\end{array}$ & $\mathrm{L}+\mathrm{D}+\mathrm{G}$ & $\mathrm{L}+\mathrm{D}+\mathrm{G}$ & 89.41 & 87.79 & 92.9 & 127.89 & 89.1 & 0.962 & 0.699 & 1.003 \\
\hline 10 & $\begin{array}{c}\text { DC7510-20-S150- } \\
\text { N-2 }\end{array}$ & $\mathrm{L}+\mathrm{D}+\mathrm{G}$ & $\mathrm{L}+\mathrm{D}+\mathrm{G}$ & 86.16 & & 90.47 & 127.44 & 86.05 & 0.952 & 0.676 & 1.001 \\
\hline 11 & $\begin{array}{c}\text { DC7510-20-S300- } \\
\text { N-1 }\end{array}$ & $\mathrm{L}+\mathrm{D}+\mathrm{G}$ & $\mathrm{L}+\mathrm{D}+\mathrm{G}$ & 87.34 & 87.10 & 91.62 & - & - & 0.953 & - & - \\
\hline 12 & $\begin{array}{c}\text { DC7510-20-S300- } \\
\text { N-2 }\end{array}$ & $\mathrm{L}+\mathrm{D}+\mathrm{G}$ & $\mathrm{L}+\mathrm{D}+\mathrm{G}$ & 86.86 & & 91.2 & - & - & 0.952 & - & - \\
\hline 13 & $\begin{array}{c}\text { DC7510-20-S150-Y- } \\
1\end{array}$ & $\mathrm{~L}+\mathrm{D}+\mathrm{G}$ & $\mathrm{L}+\mathrm{D}+\mathrm{G}$ & 87.89 & - & 92.73 & - & - & 0.937 & - & - \\
\hline 14 & $\begin{array}{c}\text { DC7510-20-S300-Y- } \\
1 \\
\end{array}$ & $\mathrm{~L}+\mathrm{D}+\mathrm{G}$ & $\mathrm{L}+\mathrm{D}+\mathrm{G}$ & 88.54 & & 92.89 & - & - & 0.953 & - & - \\
\hline 15 & $\begin{array}{c}\text { DC9008-05-S150- } \\
\text { N-1 }\end{array}$ & $\mathrm{L}+\mathrm{D}$ & $\mathrm{L}+\mathrm{D}$ & 71.03 & 69.97 & 77.72 & 105.9 & 67.88 & 0.914 & 0.671 & 1.046 \\
\hline 16 & $\begin{array}{c}\text { DC9008-05-S150- } \\
\text { N-2 }\end{array}$ & $\mathrm{L}+\mathrm{D}$ & $\mathrm{L}+\mathrm{D}$ & 68.9 & & 76.16 & 105.96 & 67.35 & 0.905 & 0.650 & 1.023 \\
\hline 17 & $\begin{array}{c}\text { DC9008-15-S150- } \\
\text { N-1 }\end{array}$ & $\mathrm{L}+\mathrm{D}$ & $\mathrm{L}+\mathrm{D}$ & 66.48 & 66.12 & 73.53 & 104.28 & 67.78 & 0.904 & 0.638 & 0.981 \\
\hline
\end{tabular}


TABle 4: Continued.

\begin{tabular}{|c|c|c|c|c|c|c|c|c|c|c|c|}
\hline No. & Specimen & $\begin{array}{c}\text { Test buckling } \\
\text { mode }\end{array}$ & $\begin{array}{c}\text { FEA buckling } \\
\text { mode }\end{array}$ & $P_{\mathrm{t}}(\mathrm{kN})$ & $\begin{array}{c}P_{\mathrm{ta}} \\
(\mathrm{kN})\end{array}$ & $P_{\mathrm{A}}(\mathrm{kN})$ & $\begin{array}{c}P_{\mathrm{D}} \\
(\mathrm{kN}) \\
\end{array}$ & $\begin{array}{l}\mathrm{P}_{\mathrm{MD}} \\
(\mathrm{kN})\end{array}$ & $\begin{array}{l}P_{\mathrm{t}} / \\
P_{\mathrm{A}} \\
\end{array}$ & $\begin{array}{l}P_{\mathrm{t}} / \\
P_{\mathrm{D}}\end{array}$ & $\begin{array}{c}P_{\mathrm{t}} / \\
\mathrm{P}_{\mathrm{MD}}\end{array}$ \\
\hline 18 & $\begin{array}{c}\text { DC9008-15-S150- } \\
\text { N-2 }\end{array}$ & $\mathrm{L}+\mathrm{D}$ & $\mathrm{L}+\mathrm{D}$ & 65.76 & & 72.06 & 103.95 & 67.23 & 0.913 & 0.633 & 0.978 \\
\hline 19 & $\begin{array}{c}\text { DC9008-15-S300- } \\
\text { N-1 }\end{array}$ & $\mathrm{L}+\mathrm{D}$ & $\mathrm{L}+\mathrm{D}$ & 67.82 & 67.31 & 74.16 & - & - & 0.915 & - & - \\
\hline 20 & $\begin{array}{c}\text { DC9008-15-S300- } \\
\mathrm{N}-2\end{array}$ & $\mathrm{~L}+\mathrm{D}$ & $\mathrm{L}+\mathrm{D}$ & 66.8 & & 73.24 & - & - & 0.912 & - & - \\
\hline 21 & $\begin{array}{c}\text { DC9008-15-S150-Y- } \\
1\end{array}$ & $\mathrm{~L}+\mathrm{D}$ & $\mathrm{L}+\mathrm{D}$ & 68.8 & - & 75.88 & - & - & 0.907 & - & - \\
\hline 22 & $\begin{array}{c}\text { DC9008-15-S300-Y- } \\
1\end{array}$ & $\mathrm{~L}+\mathrm{D}$ & $\mathrm{L}+\mathrm{D}$ & 67.9 & & 73.26 & - & - & 0.908 & - & - \\
\hline 23 & $\begin{array}{c}\text { DC9008-20-S150- } \\
\text { N-1 }\end{array}$ & $\mathrm{L}+\mathrm{D}$ & $\mathrm{L}+\mathrm{D}$ & 69.33 & 70.47 & 73.04 & 98.27 & 64.19 & 0.949 & 0.706 & 1.080 \\
\hline 24 & $\begin{array}{c}\text { DC9008-20-S150- } \\
\text { N-2 }\end{array}$ & $\mathrm{L}+\mathrm{D}$ & $\mathrm{L}+\mathrm{D}$ & 71.6 & & 73.77 & 98.05 & 65.45 & 0.971 & 0.730 & 1.094 \\
\hline 25 & $\begin{array}{c}\text { DC9008-20-S300- } \\
\text { N-1 }\end{array}$ & $\mathrm{L}+\mathrm{D}$ & $\mathrm{L}+\mathrm{D}$ & 70.16 & 70.20 & 70.42 & - & - & 0.996 & - & - \\
\hline 26 & $\begin{array}{c}\text { DC9008-20-S300- } \\
\text { N-2 }\end{array}$ & $\mathrm{L}+\mathrm{D}$ & $\mathrm{L}+\mathrm{D}$ & 70.24 & & 70.95 & - & - & 0.990 & - & - \\
\hline 27 & $\begin{array}{c}\text { DC9008-20-S150-Y- } \\
1\end{array}$ & $\mathrm{~L}+\mathrm{D}$ & $\mathrm{L}+\mathrm{D}$ & 71.21 & - & 73.23 & - & - & 0.945 & - & - \\
\hline 28 & $\begin{array}{c}\text { DC9008-20-S300-Y- } \\
1\end{array}$ & $\mathrm{~L}+\mathrm{D}$ & $\mathrm{L}+\mathrm{D}$ & 70.84 & & 72.13 & - & - & 0.941 & - & - \\
\hline 29 & $\begin{array}{c}\text { DC10008-05-S150- } \\
\text { N-1 }\end{array}$ & $\mathrm{D}+\mathrm{L}$ & $\mathrm{D}+\mathrm{L}$ & 58.29 & 59.37 & 63.32 & 71.06 & 47.61 & 0.921 & 0.820 & 1.224 \\
\hline 30 & $\begin{array}{c}\text { DC10008-05-S150- } \\
\text { N-2 }\end{array}$ & $\mathrm{D}+\mathrm{L}$ & $\mathrm{D}+\mathrm{L}$ & 60.45 & & 62.5 & 70.98 & 47.85 & 0.967 & 0.852 & 1.263 \\
\hline 31 & $\begin{array}{c}\text { DC10008-15-S150- } \\
\text { N-1 }\end{array}$ & $\mathrm{D}+\mathrm{L}$ & $\mathrm{D}+\mathrm{L}$ & 56.275 & 54.00 & 55.11 & 70.96 & 47.68 & 1.021 & 0.793 & 1.180 \\
\hline 32 & $\begin{array}{l}\text { DC10008-15-S150- } \\
\text { N-2 }\end{array}$ & $\mathrm{D}+\mathrm{L}$ & $\mathrm{D}+\mathrm{L}$ & 51.725 & & 54 & 70.75 & 47.75 & 0.958 & 0.731 & 1.083 \\
\hline 33 & $\begin{array}{c}\text { DC10008-15-S300- } \\
\text { N-1 }\end{array}$ & $\mathrm{D}+\mathrm{L}$ & $\mathrm{D}+\mathrm{L}$ & 52.6 & 52.70 & 55.23 & - & - & 0.952 & - & - \\
\hline 34 & $\begin{array}{c}\text { DC10008-15-S300- } \\
\text { N-2 }\end{array}$ & $\mathrm{D}+\mathrm{L}$ & $\mathrm{D}+\mathrm{L}$ & 52.8 & & 53.93 & - & - & 0.979 & - & - \\
\hline 35 & $\begin{array}{c}\text { DC10008-15-S150- } \\
\text { Y-1 }\end{array}$ & $\mathrm{D}+\mathrm{L}$ & $\mathrm{D}+\mathrm{L}$ & 55.7 & - & 55.19 & - & - & 1.009 & - & - \\
\hline 36 & $\begin{array}{c}\text { DC10008-15-S300- } \\
\text { Y-1 }\end{array}$ & $\mathrm{D}+\mathrm{L}$ & $\mathrm{D}+\mathrm{L}$ & 57 & & 55.22 & - & - & 1.032 & - & - \\
\hline 37 & $\begin{array}{c}\text { DC10008-20-S150- } \\
\text { N-1 }\end{array}$ & $\mathrm{D}+\mathrm{L}$ & $\mathrm{D}+\mathrm{L}$ & 56.33 & 55.57 & 54.48 & 70.65 & 47.76 & 1.034 & 0.797 & 1.179 \\
\hline 38 & $\begin{array}{c}\text { DC10008-20-S150- } \\
\text { N-2 }\end{array}$ & $\mathrm{D}+\mathrm{L}$ & $\mathrm{D}+\mathrm{L}$ & 54.8 & & 54 & 70.12 & 47.95 & 1.015 & 0.782 & 1.143 \\
\hline 39 & $\begin{array}{c}\text { DC10008-20-S300- } \\
\text { N-1 }\end{array}$ & $\mathrm{D}+\mathrm{L}$ & $\mathrm{D}+\mathrm{L}$ & 52.54 & 53.15 & 52.61 & - & - & 0.999 & - & - \\
\hline 40 & $\begin{array}{c}\text { DC10008-20-S300- } \\
\text { N-2 }\end{array}$ & $\mathrm{D}+\mathrm{L}$ & $\mathrm{D}+\mathrm{L}$ & 53.76 & & 53.14 & - & - & 1.012 & - & - \\
\hline 41 & $\begin{array}{c}\text { DC10008-20-S150- } \\
\text { Y-1 }\end{array}$ & $\mathrm{D}+\mathrm{L}$ & $\mathrm{D}+\mathrm{L}$ & 55.88 & - & 55.12 & - & - & 1.014 & - & - \\
\hline 42 & $\begin{array}{c}\text { DC10008-20-S300- } \\
\text { Y-1 }\end{array}$ & $\mathrm{D}+\mathrm{L}$ & $\mathrm{D}+\mathrm{L}$ & 53.85 & & 52.1 & - & - & 1.018 & - & - \\
\hline 43 & $\begin{array}{c}\text { DC8008-05-S150- } \\
\text { N-1 }\end{array}$ & $\mathrm{D}+\mathrm{L}$ & $\mathrm{D}+\mathrm{L}$ & 64.26 & 63.49 & 68.37 & 79.01 & 58.46 & 0.940 & 0.813 & 1.099 \\
\hline 44 & $\begin{array}{c}\text { DC8008-05-S150- } \\
\text { N-2 }\end{array}$ & $\mathrm{D}+\mathrm{L}$ & $\mathrm{D}+\mathrm{L}$ & 62.72 & & 67.67 & 78.32 & 58.12 & 0.927 & 0.801 & 1.079 \\
\hline 45 & $\begin{array}{c}\text { DC8008-15-S150- } \\
\text { N-1 }\end{array}$ & $\mathrm{L}+\mathrm{D}$ & $\mathrm{L}+\mathrm{D}$ & 55.7 & 57.50 & 55.63 & 79.71 & 57.21 & 1.001 & 0.699 & 0.974 \\
\hline 46 & $\begin{array}{c}\text { DC8008-15-S150- } \\
\text { N-2 }\end{array}$ & $\mathrm{L}+\mathrm{D}$ & $\mathrm{L}+\mathrm{D}$ & 59.3 & & 56.75 & 79.53 & 56.35 & 1.045 & 0.746 & 1.052 \\
\hline
\end{tabular}


TABle 4: Continued.

\begin{tabular}{|c|c|c|c|c|c|c|c|c|c|c|c|}
\hline No. & Specimen & $\begin{array}{c}\text { Test buckling } \\
\text { mode }\end{array}$ & $\begin{array}{l}\text { FEA buckling } \\
\text { mode }\end{array}$ & $P_{\mathrm{t}}(\mathrm{kN})$ & $\begin{array}{c}P_{\mathrm{ta}} \\
(\mathrm{kN})\end{array}$ & $P_{\mathrm{A}}(\mathrm{kN})$ & $\begin{array}{c}P_{\mathrm{D}} \\
(\mathrm{kN}) \\
\end{array}$ & $\begin{array}{l}\mathrm{P}_{\mathrm{MD}} \\
(\mathrm{kN})\end{array}$ & $\begin{array}{l}P_{\mathrm{t}} / \\
P_{\mathrm{A}} \\
\end{array}$ & $\begin{array}{l}P_{\mathrm{t}} / \\
P_{\mathrm{D}} \\
\end{array}$ & $\begin{array}{c}P_{\mathrm{t}} / \\
\mathrm{P}_{\mathrm{MD}}\end{array}$ \\
\hline 47 & $\begin{array}{c}\text { DC8008-15-S300- } \\
\text { N-1 }\end{array}$ & $\mathrm{L}+\mathrm{D}$ & $\mathrm{L}+\mathrm{D}$ & 54.4 & 55.90 & 53.43 & - & - & 1.018 & - & - \\
\hline 48 & $\begin{array}{c}\text { DC8008-15-S300- } \\
\text { N-2 }\end{array}$ & $\mathrm{L}+\mathrm{D}$ & $\mathrm{L}+\mathrm{D}$ & 57.4 & & 55.04 & - & - & 1.043 & - & - \\
\hline 49 & $\begin{array}{c}\text { DC8008-15-S150-Y- } \\
1\end{array}$ & $\mathrm{~L}+\mathrm{D}$ & $\mathrm{L}+\mathrm{D}$ & 57.96 & - & 53.35 & - & - & 0.955 & - & - \\
\hline 50 & $\begin{array}{c}\text { DC8008-15-S300-Y- } \\
1\end{array}$ & $\mathrm{~L}+\mathrm{D}$ & $\mathrm{L}+\mathrm{D}$ & 59.1 & & 59.74 & - & - & 0.989 & - & - \\
\hline 51 & $\begin{array}{c}\text { DC8008-20-S150- } \\
\text { N-1 }\end{array}$ & $\mathrm{L}+\mathrm{D}+\mathrm{G}$ & $\mathrm{L}+\mathrm{D}+\mathrm{G}$ & 57.97 & 56.40 & 54.68 & 79.03 & 55.13 & 1.060 & 0.734 & 1.052 \\
\hline 52 & $\begin{array}{l}\text { DC8008-20-S150- } \\
\text { N-2 }\end{array}$ & $\mathrm{L}+\mathrm{D}+\mathrm{G}$ & $\mathrm{L}+\mathrm{D}+\mathrm{G}$ & 54.82 & & 54.21 & 78.21 & 54.45 & 1.011 & 0.701 & 1.007 \\
\hline 53 & $\begin{array}{c}\text { DC8008-20-S300- } \\
\text { N-1 }\end{array}$ & $\mathrm{L}+\mathrm{D}+\mathrm{G}$ & $\mathrm{L}+\mathrm{D}+\mathrm{G}$ & 52.5 & 52.04 & 53.31 & - & - & 0.985 & - & - \\
\hline 54 & $\begin{array}{c}\text { DC8008-20-S300- } \\
\text { N-2 }\end{array}$ & $\mathrm{L}+\mathrm{D}+\mathrm{G}$ & $\mathrm{L}+\mathrm{D}+\mathrm{G}$ & 51.58 & & 52.24 & - & - & 0.987 & - & - \\
\hline 55 & $\begin{array}{c}\text { DC8008-20-S150-Y- } \\
1\end{array}$ & $\mathrm{~L}+\mathrm{D}+\mathrm{G}$ & $\mathrm{L}+\mathrm{D}+\mathrm{G}$ & 57.14 & - & 55.48 & - & - & 0.976 & - & - \\
\hline 56 & $\begin{array}{c}\text { DC8008-20-S300-Y- } \\
1\end{array}$ & $\mathrm{~L}+\mathrm{D}+\mathrm{G}$ & $\mathrm{L}+\mathrm{D}+\mathrm{G}$ & 52.73 & & 53.66 & - & - & 0.964 & - & - \\
\hline \multicolumn{9}{|c|}{ Average } & 0.969 & 0.744 & 1.066 \\
\hline \multirow{2}{*}{\multicolumn{9}{|c|}{$\begin{array}{l}\text { Standard deviation } \\
\text { Coefficient of variation }\end{array}$}} & 0.036 & 0.057 & 0.064 \\
\hline & & & & & & & & & 0.037 & 0.077 & 0.060 \\
\hline
\end{tabular}

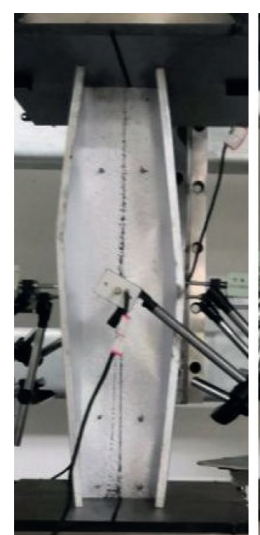

DC10008-05

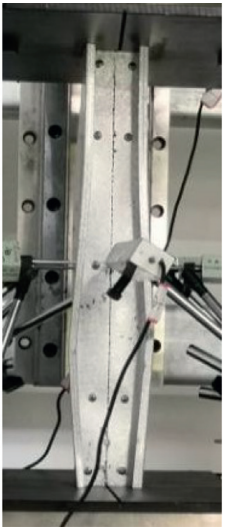

DC7510-05

(a)

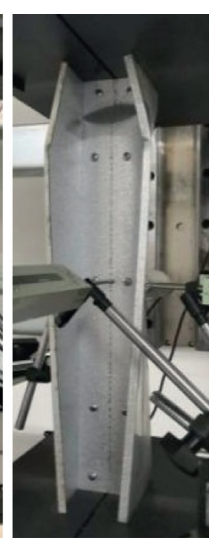

DC8008-05

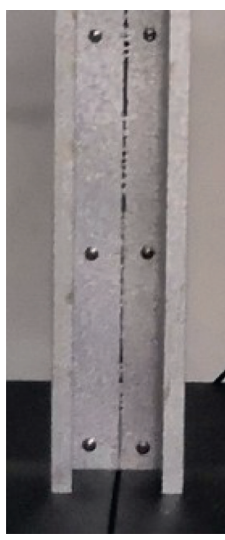

DC9008-05

(b)

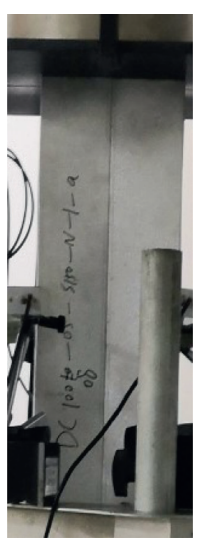

DC10008-05

(c)

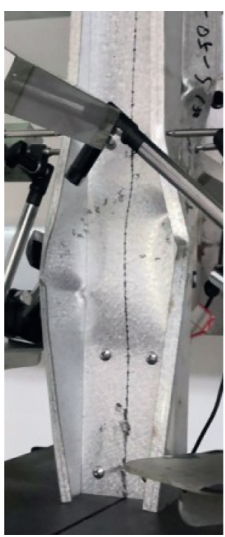

DC7510-05

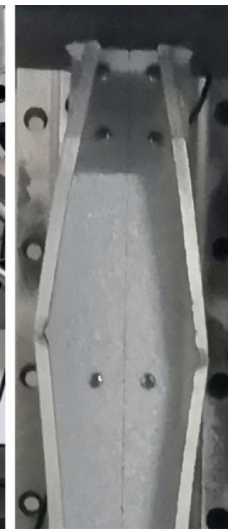

DC9008-05

(d)

Figure 9: Buckling modes of built-up columns with the nominal length of $500 \mathrm{~mm}$. (a) Distortional buckling + local buckling in web. (b) Local buckling in web. (c) Local buckling in the flange. (d) Plastic crush.

$P_{\text {ta }}$ were the tested ultimate load and the average ultimate capacities of the two repeated specimens. From Table 4, it can be known that for section DC7510 series and section DC8008 series, the buckling modes changed from the interaction of local and distortional buckling to the interaction of local, distortional, and overall buckling. Meanwhile, the length had significant effect on ultimate strength of specimens failed with the interaction of local, distortional, and overall buckling because the load capacities of these specimens decreased obviously with the increasing of length. But, for the section DC9008 series and section DC10008 series, all specimens failed with the interaction of local and distortional buckling, and the ultimate load capacities had no obvious change which indicated that the length had less influence on ultimate strength of specimens failed with the interaction of local and distortional buckling.

The ultimate load capacities increased $2 \%-5 \%$ when the spacing of screws changed from $300 \mathrm{~mm}$ to $150 \mathrm{~mm}$. The results indicated the reducing the spacing of connection screws for same section can result in a certain increase in the ultimate load capacity of the specimen.

The ultimate load capacities increased 3\%-10\% when the end fastener group was installed. The results indicated that the arrangement of EFG for the same section can lead 


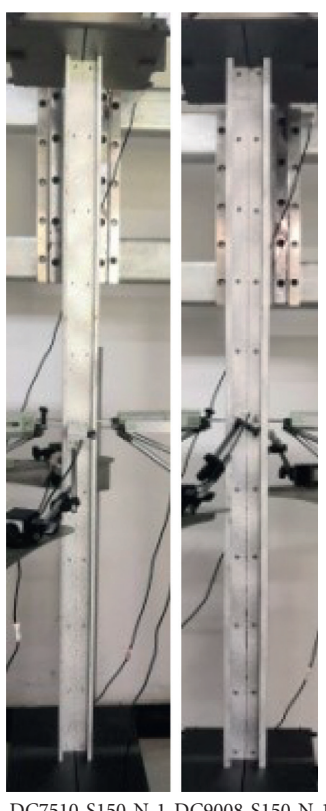

(a)

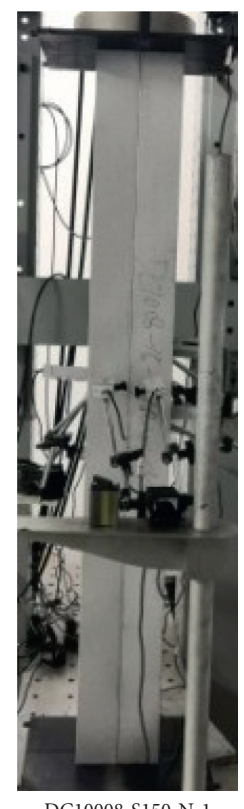

(b)

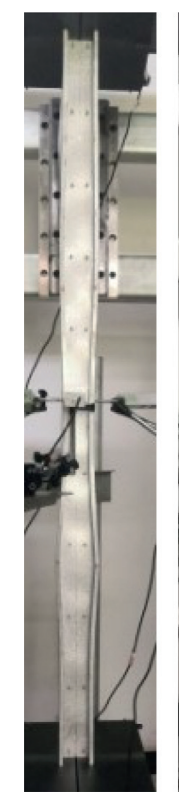

DC7510-S150-N-1 DC8008-S150-N-1

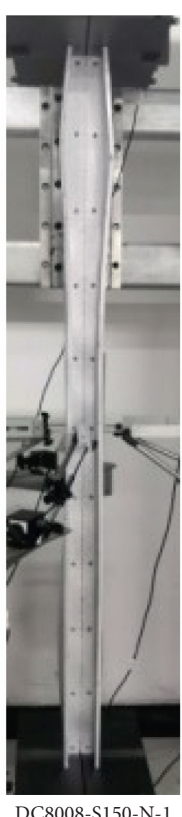

(c)

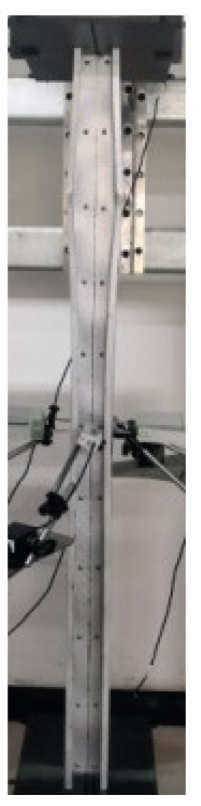

DC9008-S150-N-1

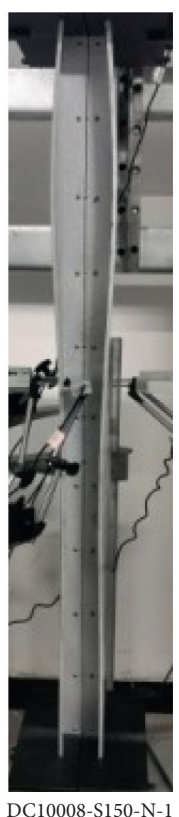

DC10008-S150-N-1

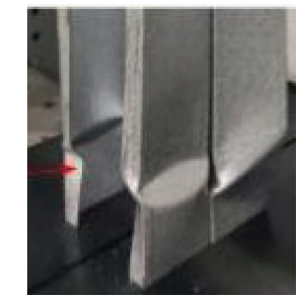

(d)

Figure 10: Buckling modes of built-up columns with the nominal length of $1500 \mathrm{~mm}$. (a) Local buckling in web. (b) Local buckling in the flange. (c) Distortional buckling + local buckling in web. (d) Plastic crush.

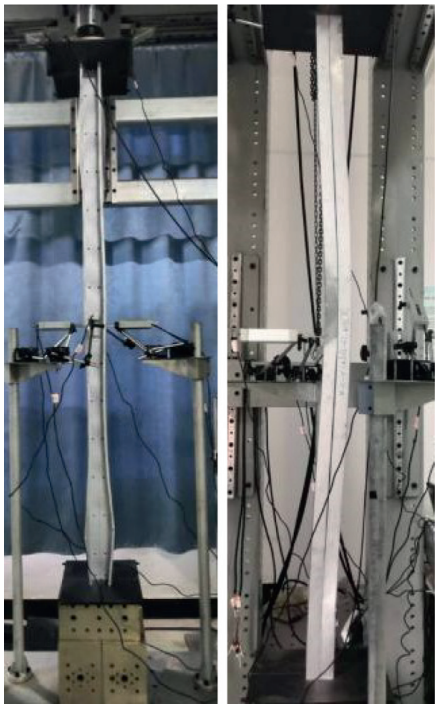

DC7510

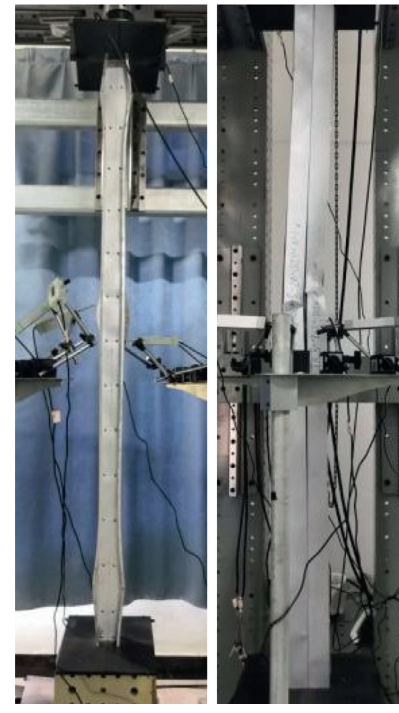

DC8008

(a)

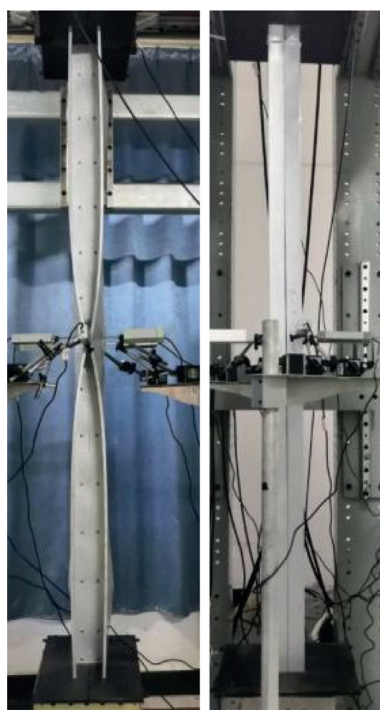

DC9008

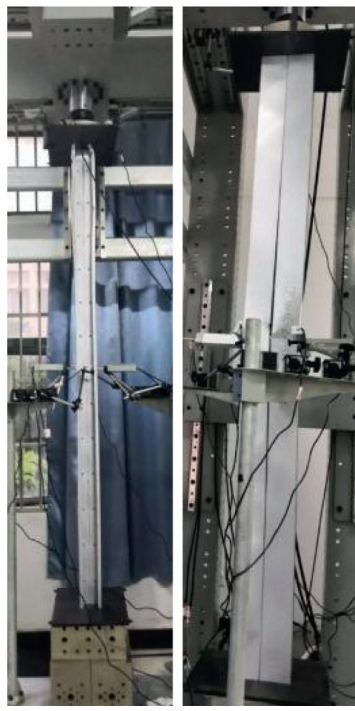

DC10008

(b)

Figure 11: Buckling modes of built-up columns with the nominal length of $2000 \mathrm{~mm}$. (a) Local buckling in web + distortional buckling + overall flexural buckling. (b) Local buckling in web + local buckling in flange + distortional buckling.

to a certain increase in the ultimate strength of the specimen.

\section{Verification of FEM and Parametric Study}

4.1. Development of Finite Element Models. The finite element program ABAQUS [29] was used to simulate the buckling modes and behavior of the specimens and perform a further parametric analysis on the built-up I-sectional columns. The S4R shell element was selected for modeling the column, and the analytical rigid body element was used for modeling the upper and lower end steel plates of the specimens. The C3D8R solid element was used to simulate the ST4.8 self-drilling screws used in the test. The selfdrilling screw and the web were connected by tie (binding). Because the two web surfaces were prone to puncture in the FEA, the hard contact between the contact surfaces of the webs was used to transfer the normal stress between the two 


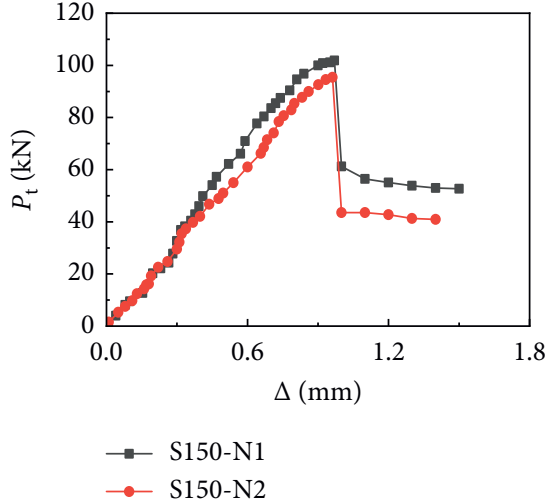

(a)

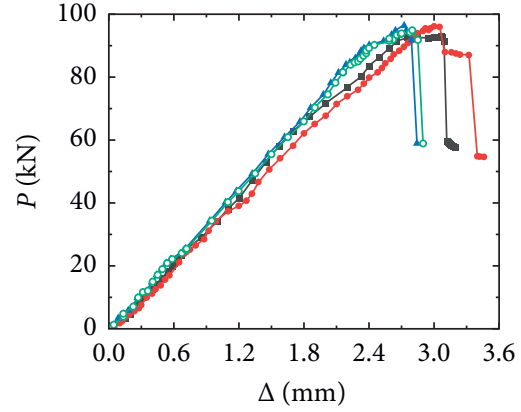

$\rightarrow \mathrm{S} 150-\mathrm{N} 1 \rightarrow \mathrm{S} 150-\mathrm{Y} 1$
$\rightarrow \mathrm{S} 300-\mathrm{N} 2 \rightarrow \mathrm{S} 300-\mathrm{Y} 1$

(b)
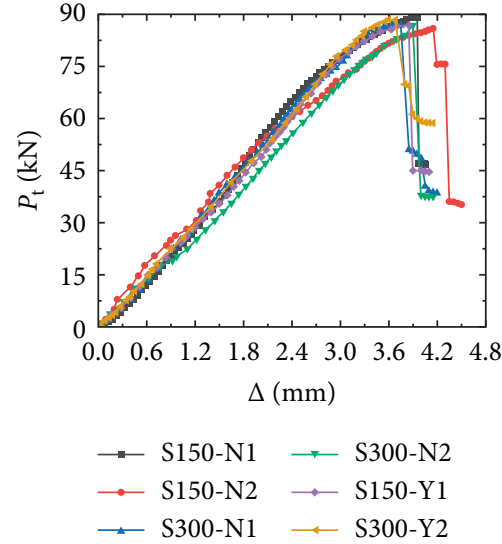

(c)

FIGURE 12: Load and axial shorten displacement curve of section DC7510 series. (a) Section DC7510-05 series. (b) Section DC7510-15 series. (c) Section DC7510-20 series.

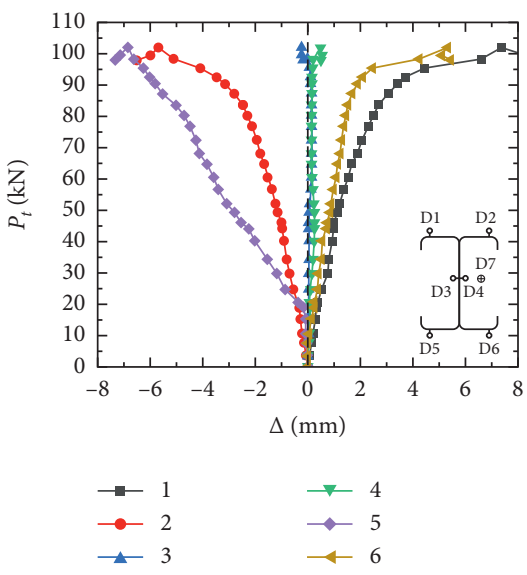

(a)

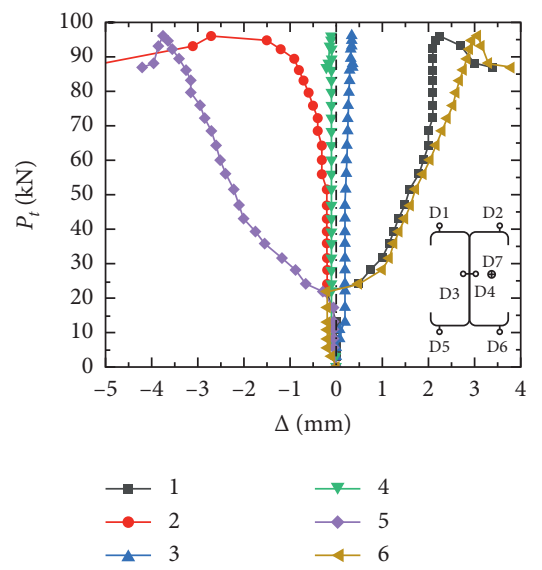

(b)

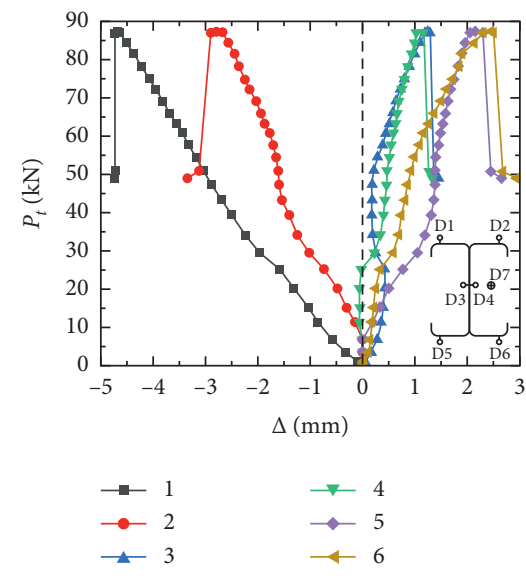

(c)

Figure 13: Load and lateral displacement curve of section DC7510 series. (a) DC7510-05-S150-N-1. (b) DC7510-15-S150-Y-1. (c) DC751020-S150-N-1.

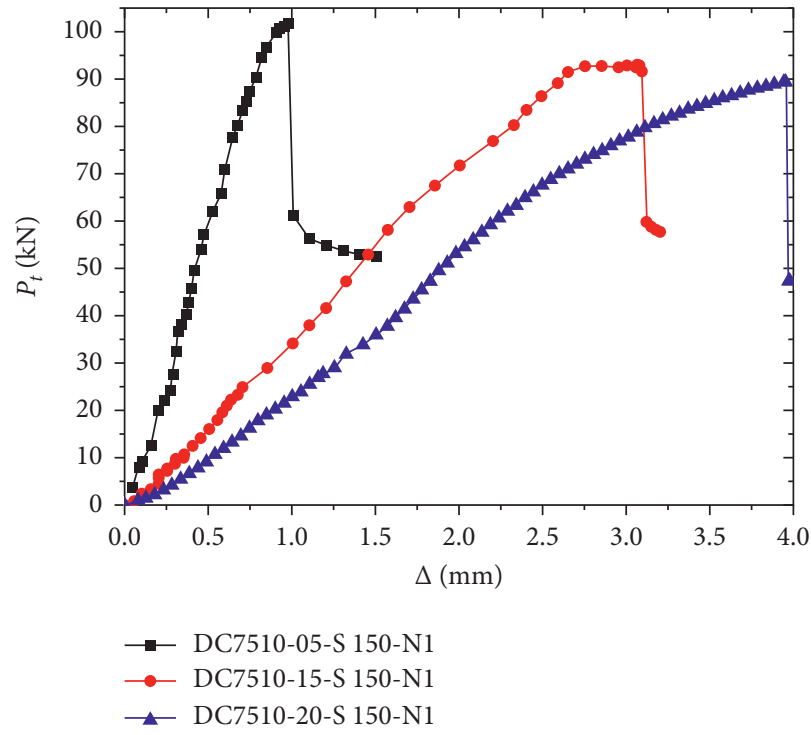

FIGURE 14: Load and axial shorten displacement curve of section DC7510 series with different lengths. 
surfaces. The end constraint conditions of the test plate were all fixed, by constraining the five degrees of freedom of the reference point RP1 at the upper end plate ( 2 translation degrees of freedom and 3 rotational degree of freedom, releasing UZ degrees of freedom to control displacement) and the six degrees of freedom of the reference point RP2 at the lower end plate. The measured actual dimensions and the maximum initial imperfections of the specimens (see in Table 2) were all included in the models. The ideal elasticplastic constitutive was selected based on the coupon tests (see in Table 3). The whole analysis procedure included two phases: firstly, an eigen-value buckling analysis was conducted to find the first elastic buckling mode of the specimen. The measured maximum magnitude of the initial imperfections was applied to the first eigen mode to produce the geometric imperfection of FEA. Secondly, nonlinear analysis considering both the material nonlinear and geometric nonlinear was performed by using the arc-length method to analyze the failure modes and load capacities of specimens. The final FEM is shown in Figure 15.

4.2. Verification of Finite Element Models. The comparisons on the ultimate strengths and the buckling modes between test and FEA results are summarized in Table 4 , where $P_{\mathrm{A}}$ represents the ultimate strengths of specimens obtained by FEA. The mean value of the ratio of test results to FEA results $\left(P_{\mathrm{t}} / P_{\mathrm{A}}\right)$ is 0.969 with the corresponding coefficient of variation of 0.037 . It shows that the FEA results match well with the test results in the ultimate strengths. So, it indicates that the FEA can simulate the ultimate strengths of specimens accurately. The comparisons on failure modes for the section DC10008 series between the test and FEA are illustrated in Figure 16, and the interaction of distortional buckling and local buckling can be seen. The comparisons show good agreement about buckling modes between the test and FEA, which indicates that the FEM can analyze the buckling modes of this kind of built-up column well. The load and axial shorten displacement curves of section DC9008 obtained by the FEA coincide well with the experimental result, as shown in Figures 17(a)-17(c). Meanwhile, the comparison on load and lateral displacement curves of specimen DC7510-15-S150-Y-1 shown in Figure 17(d) indicated a good agree about buckling mode, but the displacement values had a litter different. Therefore, the FEM verified by the tests is accurate and can be used to simulate the ultimate strength, the failure mode, and the load and axial displacement curve of CFS built-up I-sectional columns.

4.3. Finite Element Parametric Study. The main parametric studies in finite element analysis were the slenderness ratio, the spacing of screws, and the end fastener group. The nominal dimension of cross section and the ideal elasticplastic constitutive relation were used in FEM. The maximum magnitude of the initial geometric imperfection supposed to be $L / 750$ according to the Chinese specification [1]. The other conditions such as restraint conditions in the FEM were same as introduced in Section 4.1.

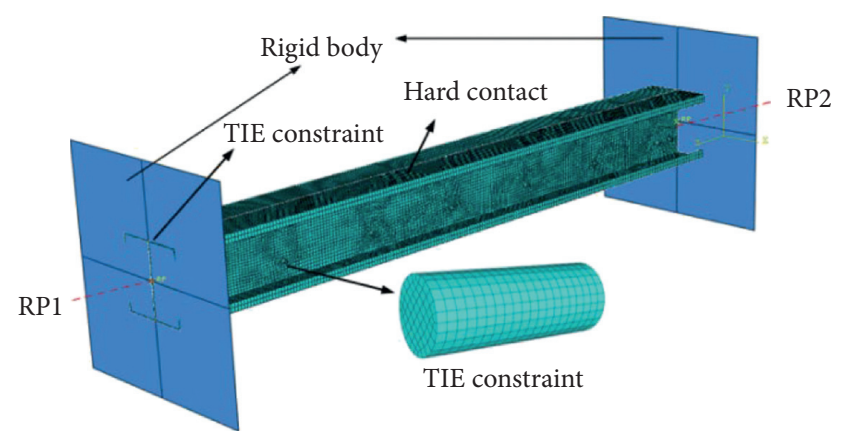

Figure 15: Finite element analysis model.

4.3.1. Effect of the Slenderness Ratio. The two tested and six other normal sections were studied further by using program ABAQUS in the same way. The nominal dimensions of cross sections selected to analyze are listed in Table 5. The lengths of chosen built-up columns were 500, 1000, 1500, 2000 , and $3000 \mathrm{~mm}$. The spacing of screws was $150 \mathrm{~mm}$. The maximum percent ratios of decreasing of load capacities with the slenderness ratio are listed in Table 5. The load capacities obtained by FEA results are plotted in Figure 18. Meanwhile, the load-axial displacement curves of built-up I-sectional columns with different slenderness ratios and yield strengths are illustrated in Figure 18. The typical buckling modes are shown in Figure 19 for section DC7510 series.

It can be observed from Figures 18 and 19 that the ultimate strengths of the CFS built-up I-sectional columns decrease with the increasing of the slenderness ratio of columns. Figure 20 illustrated the effect of the slenderness ratio on buckling modes. For section DC7510 with nominal length of $3000 \mathrm{~mm}$ (Figure 20(a)) and $2500 \mathrm{~mm}$ (Figure 20(b)), the overall buckling is observed and the deformation of cross section does not display the local and distortional buckling. For section DC7510 with nominal length of $2000 \mathrm{~mm}$ (Figure 20(c)) and $1500 \mathrm{~mm}$ (Figure 20(d)), the overall buckling is observed and the deformation of cross section displays the distortional buckling. For section DC7510 with nominal length of $1000 \mathrm{~mm}$ (Figure 20(e)) and $500 \mathrm{~mm}$ (Figure 20(f)), the deformations of members show the interaction of overall buckling, distortional buckling, and local of flanges.

4.3.2. Effect of the Spacing of Screws. The effects of the spacing of screws were also numerically investigated. The load and axial displacement curves of section DC10010 with different lengths $(1000,2000$, and $3000 \mathrm{~mm})$ and different spacing of screws $(150,300$, and $450 \mathrm{~mm})$ are depicted in Figure 21. Columns failed by combining overall, local, and distortional buckling modes in different ways, depending of the slenderness ratio related to each of the modes. The decreasing of load capacities is less than $5 \%$ when the spacing of screws increases from $150 \mathrm{~mm}$ to $450 \mathrm{~mm}$, as shown in Figure 20. The comparison results indicate that spacing of screws has a litter effect on the ultimate strength of built-up I-sectional columns when the spacing of screws is less than $450 \mathrm{~mm}$. 


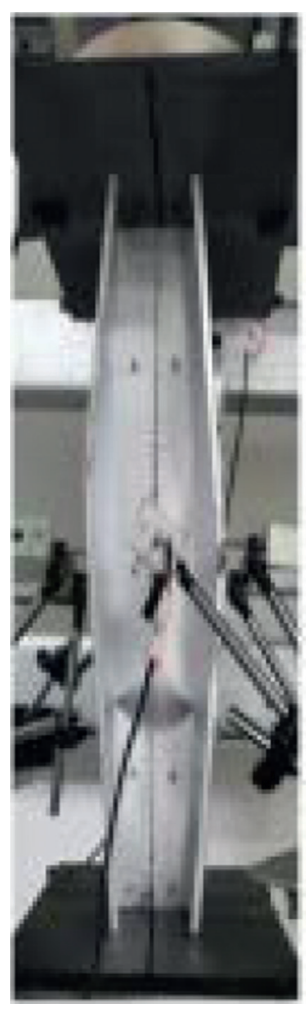

(a)
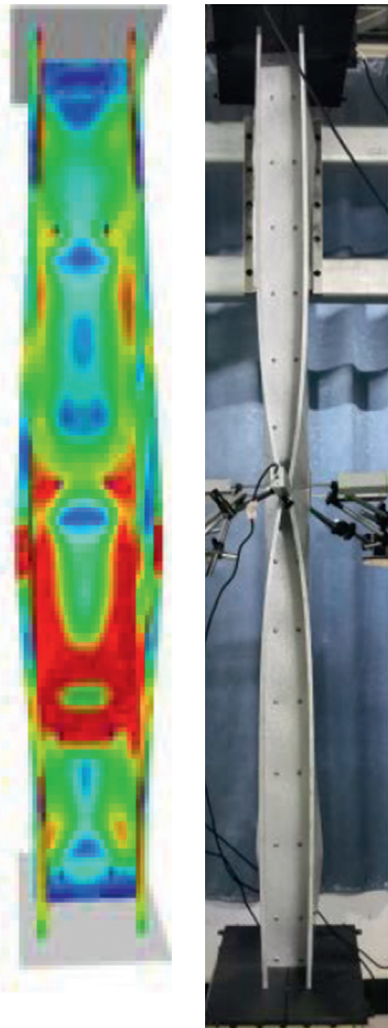

(b)

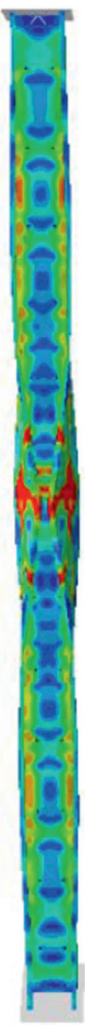

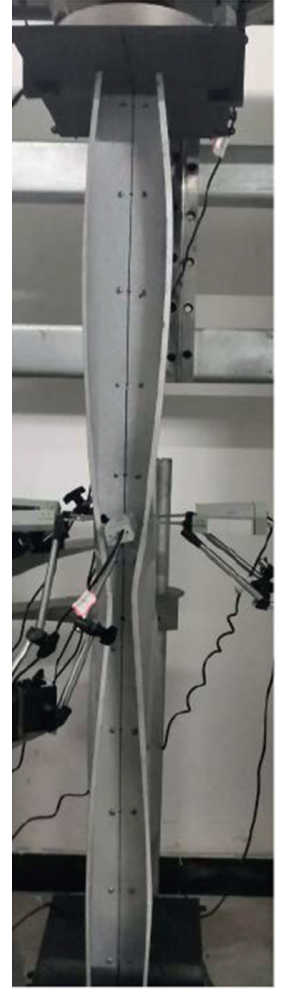

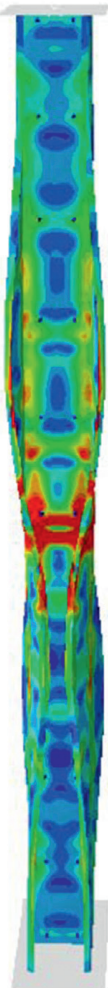

(c)

FIGURE 16: Comparison of buckling modes between tests and finite element analysis. (a) DC10008-05. (b) DC10008-15. (c) DC10008-20.

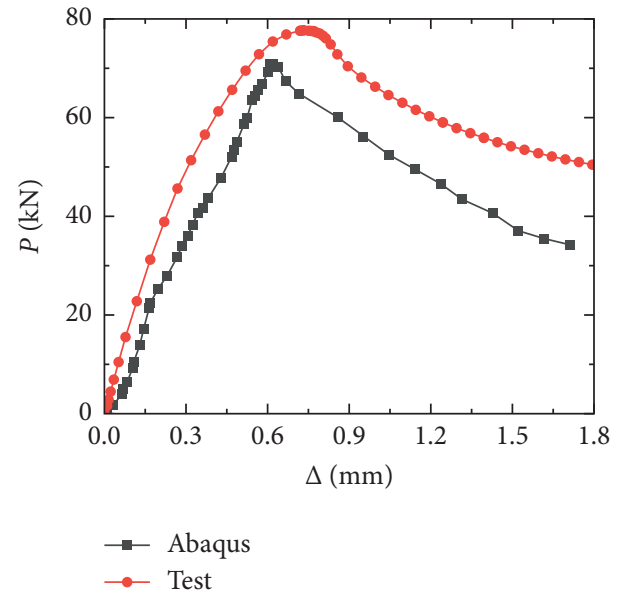

(a)

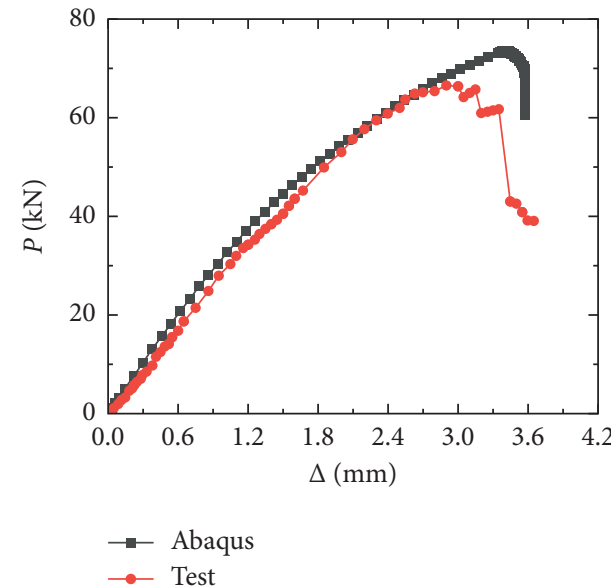

(b)

Figure 17: Continued. 


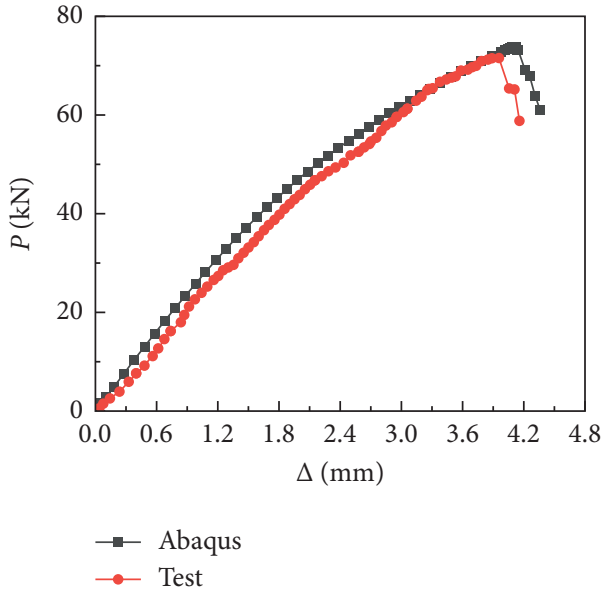

(c)

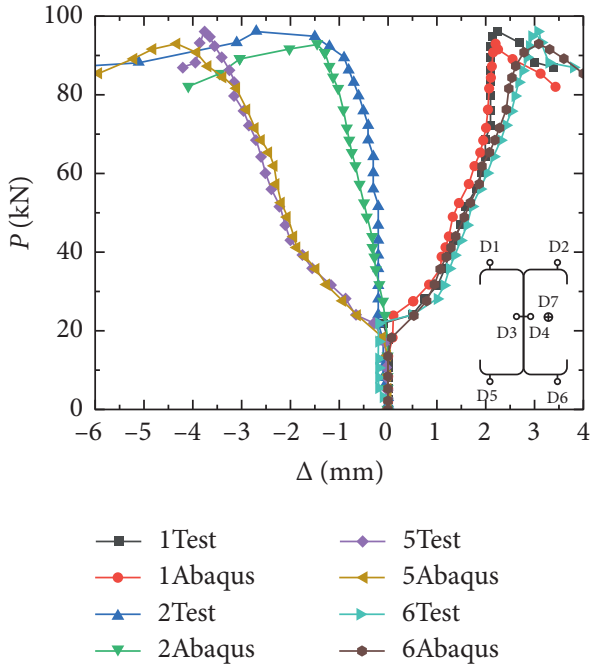

(d)

Figure 17: Comparison on load and displacement curves between tests and finite element analysis. (a) Load and axial displacement of DC9008-05-S150-N-1. (b) Load and axial displacement of DC9008-15-S150-N-1. (c) Load and axial displacement of DC9008-20-S150-N-1. (d) Load and lateral displacement of DC7510-15-S150-Y-1.

TABLE 5: Section matrix of CFS built-up columns in FEA and maximum percent ratio of decreasing of load capacity.

\begin{tabular}{lccccccc}
\hline Section series & $h(\mathrm{~mm})$ & $b(\mathrm{~mm})$ & $a(\mathrm{~mm})$ & $t(\mathrm{~mm})$ & $f_{y}(\mathrm{MPa})$ & Slenderness ratio & $\begin{array}{c}\text { Maximum percent ratio of decreasing of load capacity } \\
(\%)\end{array}$ \\
\hline DC7510 & 75 & 40 & 8 & 1 & 550 & $12.15 \sim 150.12$ & 76.72 \\
DC10010 & 100 & 51 & 12 & 1 & 550 & $9.88 \sim 118.58$ & 58.74 \\
DC10075 & 100 & 51 & 12 & 0.75 & 550 & $9.88 \sim 118.58$ & 50.11 \\
DC7012 & 69 & 35 & 11.3 & 1.2 & 350 & $13.89 \sim 166.64$ & 76.9 \\
DC14008 & 140 & 50 & 10.3 & 0.8 & 350 & $11.24 \sim 134.88$ & 48.26 \\
DC14012 & 140 & 50 & 11.3 & 1.2 & 350 & $11.24 \sim 134.88$ & 53.88 \\
DC8910 & 89 & 41 & 13 & 1 & 280 & $11.71 \sim 140.60$ & 58.89 \\
DC14010 & 140 & 41 & 13 & 1 & 280 & $13.14 \sim 157.74$ & \\
\hline
\end{tabular}

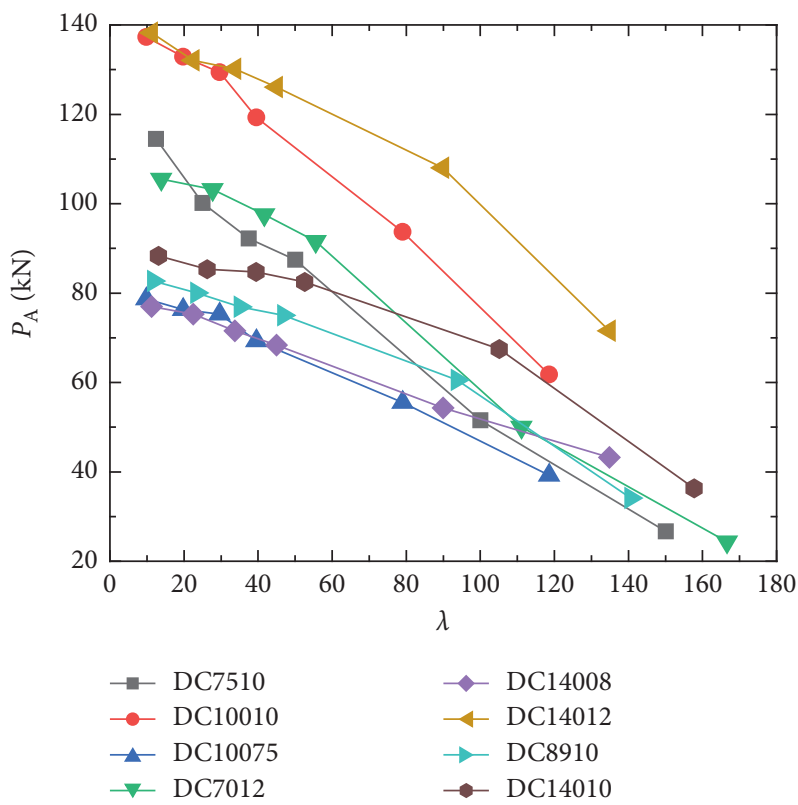

FIGURE 18: Load capacity-slenderness ratio curves of built-up I-sectional columns for different sections. 

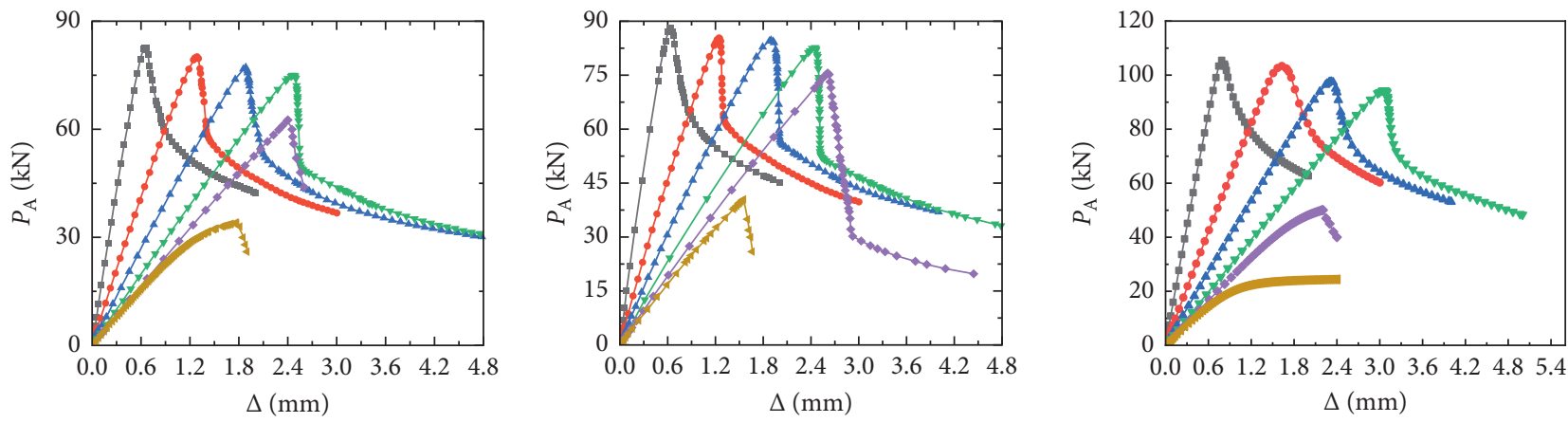

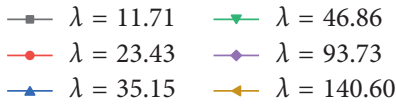

(a)

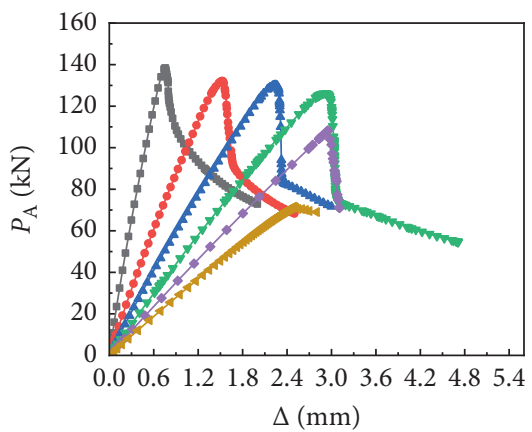

$$
\begin{aligned}
& \because \lambda=11.24 \quad \because \lambda=44.96 \\
& \because \lambda=22.48 \quad \because \lambda=89.92 \\
& \longleftarrow \lambda=33.72 \quad \longleftarrow \lambda=134.88
\end{aligned}
$$

(d)

$$
\begin{aligned}
& \multimap \lambda=13.14 \quad \because \lambda=52.58 \\
& \because \lambda=26.29 \rightarrow \lambda=105.16 \\
& \multimap \lambda=39.43 \multimap \lambda=157.74
\end{aligned}
$$

(b)

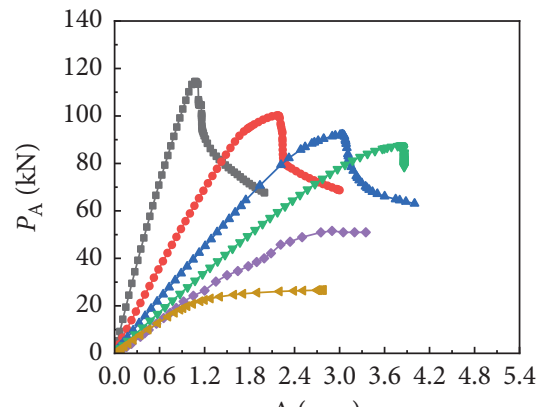

$\Delta(\mathrm{mm})$

$$
\begin{aligned}
& \because \lambda=12.5 \quad \rightarrow \lambda=50 \\
& \because \lambda=25 \quad \because \lambda=100 \\
& \longleftarrow \lambda=37.5 \longleftarrow \lambda=150
\end{aligned}
$$

(e)

$$
\begin{aligned}
& \because \lambda=13.88 \quad \longrightarrow \lambda=55.54 \\
& \because \lambda=27.77 \quad \multimap \lambda=111.09 \\
& \longleftarrow \lambda=41.66 \quad-\lambda=166.64
\end{aligned}
$$

(c)

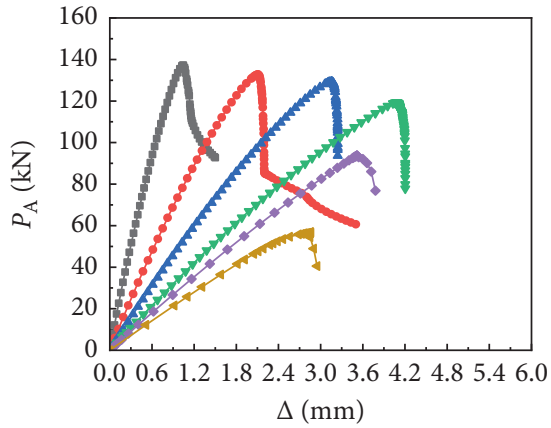

$$
\begin{aligned}
& \because \lambda=9.88 \quad \longrightarrow \lambda=39.52 \\
& \because \lambda=19.76 \multimap \lambda=79 \\
& \because \lambda=29.64 \longleftarrow \lambda=118.59
\end{aligned}
$$

(f)

FIGURE 19: Load and axial shorten displacement curves of built-up I-sectional columns with different slenderness ratios. (a) DC8910 series. (b) DC14010 series. (c) DC7012 series. (d) DC14012 series. (e) DC7510 series. (f) DC10010 series.

4.3.3. Effect of the End Fastener Group. The load capacities and buckling modes of section DC10010 and section DC7510 with and without the end fastener group are presented in Table 6, where $P_{\mathrm{A} 1}$ and $P_{\mathrm{A} 2}$ are the FEA results for built-up columns without and with EFG. The lengths of these sections were 1500 and $2000 \mathrm{~mm}$, and the spacing of screws were 150, 300, and $450 \mathrm{~mm}$. The FEA results shown in Table 6 indicated that the EFG has no obvious effect on the ultimate strength of CFS built-up I-sectional columns when the spacing of screws is equal to 150 or $300 \mathrm{~mm}$, but the EFG has a certain effect on the ultimate strength when the spacing of screws is equal to $450 \mathrm{~mm}$. The comparison results show that the EFG can increase the ultimate strength of CFS builtup I-sectional columns which fail in interaction of local and distortional buckling when the spacing of screws is equal to $450 \mathrm{~mm}$.

\section{Design Method}

The EWM and DSM are two basic design methods for CFS members in the worldwide design specifications. The DSM is only selected to determine the ultimate strength of the CFS built-up I-sectional columns in this paper. But we need to note that the current DSM has no design provision for the CFS built-up I-sectional columns. Therefore, the feasibility of DSM for determining the ultimate strength of CFS builtup I-sectional columns under axial compression should be evaluated. 


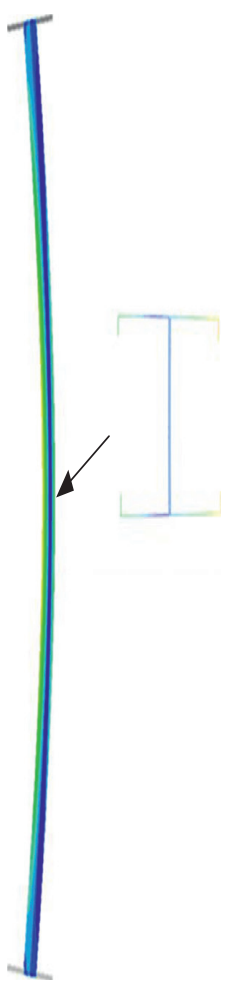

(a)

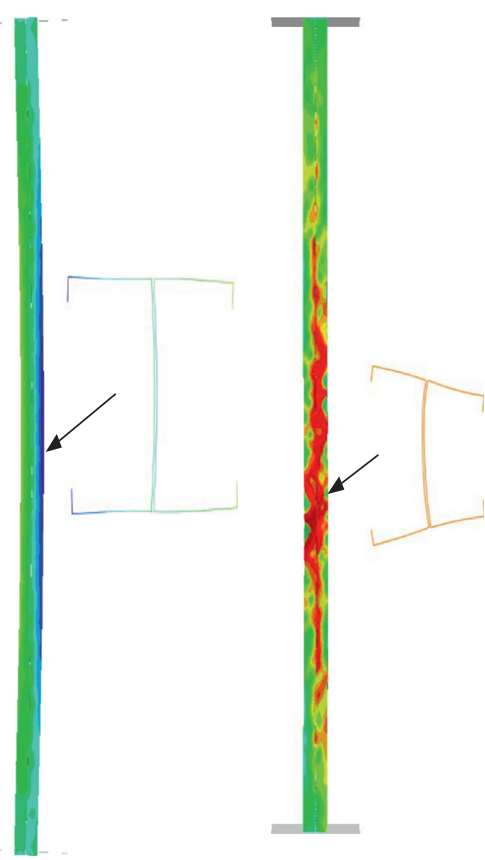

(b)

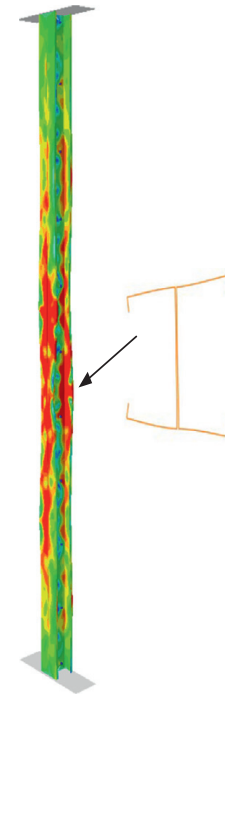

(d)
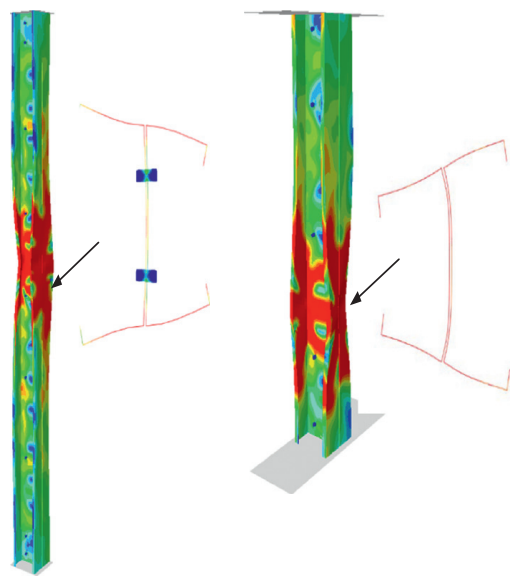

Figure 20: Failure modes of finite element analysis for section DC7510 series. (a) $3000 \mathrm{~mm}$. (b) $2500 \mathrm{~mm}$. (c) $2000 \mathrm{~mm}$. (d) $1500 \mathrm{~mm}$. (e) $1000 \mathrm{~mm}$. (f) $500 \mathrm{~mm}$.

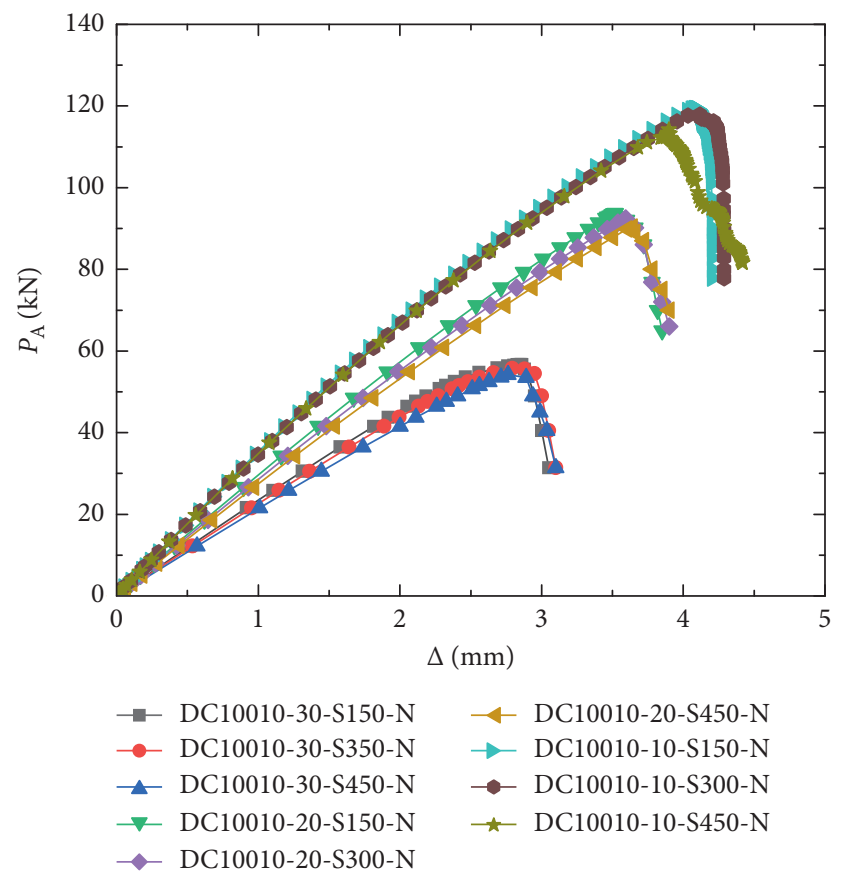

Figure 21: Load and axial displacement curves of built-up I-sectional columns with different spacing of screws.

5.1. Direct Strength Method. The DSM in AISI [2] considers the distortional buckling, the overall buckling, and the interaction of local buckling and overall buckling. The ultimate strength $\left(P_{\mathrm{D}}\right)$ of CFS columns under axial compression is equal to the minimum value of $P_{\text {ne }}, P_{\mathrm{nl}}$, and $P_{\mathrm{nd}}$ as follows:

$$
P_{\mathrm{D}}=\min \left\{P_{\mathrm{ne}}, P_{\mathrm{nl}}, P_{\mathrm{nd}}\right\} .
$$

$P_{\text {ne }}, P_{\text {nl }}$, and $P_{\text {nd }}$ can be predicted by using the following formulas:

$$
\begin{aligned}
& P_{\mathrm{ne}}= \begin{cases}\left(0.658^{\lambda_{c}^{2}}\right) P_{y}, \quad \lambda_{c} \leq 1.5, \\
\left(\frac{0.877}{\lambda_{c}^{2}}\right) P_{y}, \quad \lambda_{c}>1.5,\end{cases} \\
& P_{\mathrm{nl}}= \begin{cases}P_{\mathrm{ne}}, & \lambda_{l} \leq 0.776, \\
\left(1-0.15\left(\frac{P_{\mathrm{crl}}}{P_{\mathrm{ne}}}\right)^{0.4}\right)\left(\frac{P_{\mathrm{crl}}}{P_{\mathrm{ne}}}\right)^{0.4} P_{\mathrm{ne}}, & \lambda_{l}>0.776,\end{cases} \\
& P_{\mathrm{nd}}= \begin{cases}P_{y}, & \lambda_{d} \leq 0.561, \\
\left(1-0.25\left(\frac{P_{\mathrm{crd}}}{P_{y}}\right)^{0.6}\right)\left(\frac{P_{\mathrm{crd}}}{P_{y}}\right)^{0.6} P_{y}, & \lambda_{d}>0.561,\end{cases}
\end{aligned}
$$

where $\quad \lambda_{c}=\sqrt{P_{y} / P_{\text {cre }}}, \quad \lambda_{l}=\sqrt{P_{\mathrm{ne}} / P_{\mathrm{crl}}}, \quad \lambda_{d}=\sqrt{P_{y} / P_{\mathrm{crd}}}$, $P_{y}=A_{g} f_{y}, P_{\mathrm{crl}}=A_{g} f_{\mathrm{cl}}, P_{\mathrm{crd}}=A_{g} f_{\mathrm{cd}}, A_{g}$ is the gross 
TABLE 6: Comparison on load capacities for built-up columns with and without the end fastener group.

\begin{tabular}{|c|c|c|c|c|c|}
\hline Built-up columns & $L(\mathrm{~mm})$ & $\lambda$ & Buckling modes & $P_{\mathrm{A}}(\mathrm{kN})$ & $\left(P_{\mathrm{A} 2}-P_{\mathrm{A} 1}\right) / P_{\mathrm{A} 1}(\%)$ \\
\hline $\begin{array}{l}\text { DC10010-15-S150-N-S } \\
\text { DC10010-15-S150-Y-S } \\
\text { DC10010-15-S300-N-S } \\
\text { DC10010-15-S300-Y-S } \\
\text { DC10010-15-S450-N-S } \\
\text { DC10010-15-S450-Y-S }\end{array}$ & 1500 & 29.65 & $\begin{array}{l}L+D \\
L+D \\
L+D \\
L+D \\
L+D \\
L+D\end{array}$ & $\begin{array}{l}129.40 \\
129.60 \\
127.51 \\
128.32 \\
122.80 \\
127.80 \\
\end{array}$ & 0.64 \\
\hline $\begin{array}{l}\text { DC10010-20-S150-N-S } \\
\text { DC10010-20-S150-Y-S } \\
\text { DC10010-20-S300-N-S } \\
\text { DC10010-20-S300-Y-S } \\
\text { DC10010-20-S450-N-S } \\
\text { DC10010-20-S450-Y-S }\end{array}$ & 2000 & 39.53 & $\begin{array}{l}\mathrm{L}+\mathrm{D} \\
\mathrm{L}+\mathrm{D} \\
\mathrm{L}+\mathrm{D} \\
\mathrm{L}+\mathrm{D} \\
\mathrm{L}+\mathrm{D} \\
\mathrm{L}+\mathrm{D}\end{array}$ & $\begin{array}{l}119.29 \\
120.24 \\
117.92 \\
120.46 \\
113.20 \\
119.47 \\
\end{array}$ & $\begin{array}{l}2.15 \\
5.54\end{array}$ \\
\hline $\begin{array}{l}\text { DC7510-15-S150-N-S } \\
\text { DC7510-15-S150-Y-S } \\
\text { DC7510-15-S300-N-S } \\
\text { DC7510-15-S300-Y-S } \\
\text { DC7510-15-S450-N-S } \\
\text { DC7510-15-S450-Y-S }\end{array}$ & 1500 & 37.53 & $\begin{array}{l}L+D \\
L+D \\
L+D \\
L+D \\
L+D \\
L+D\end{array}$ & $\begin{array}{c}92.21 \\
93.58 \\
91.3 \\
92.6 \\
87.63 \\
91.5\end{array}$ & $\begin{array}{l}1.42 \\
4.42\end{array}$ \\
\hline $\begin{array}{l}\text { DC7510-20-S150-N-S } \\
\text { DC7510-20-S150-Y-S } \\
\text { DC7510-20-S300-N-S } \\
\text { DC7510-20-S300-Y-S } \\
\text { DC7510-20-S450-N-S } \\
\text { DC7510-20-S450-Y-S }\end{array}$ & 2000 & 50.04 & $\begin{array}{l}L+D \\
L+D \\
L+D \\
L+D \\
L+D \\
L+D\end{array}$ & $\begin{array}{c}87.43 \\
89.92 \\
86.68 \\
88.76 \\
82.8 \\
87.6\end{array}$ & 2.40 \\
\hline
\end{tabular}

cross-sectional area, $P_{\text {ne }}, P_{\text {nl }}$, and $P_{\text {nd }}$ are the nominal strength for overall buckling, interaction of local and global buckling, and distortional buckling, respectively, $P_{\text {cre }}, P_{\mathrm{crl}}$, and $P_{\text {crd }}$ are the elastic critical load for overall, local, distortional buckling, respectively, and $f_{\text {cre }}, f_{\text {crl }}$, and $f_{\text {crd }}$ are the elastic critical stress for overall, local, and distortional buckling, respectively.

The prediction of the elastic critical overall, local, and distortional buckling stress $\left(f_{\text {cre }}, f_{\text {crl }}\right.$, and $\left.f_{\text {crd }}\right)$ is the key content before determining the ultimate strength of the CFS members by using DSM. The elastic critical overall, local, and distortional buckling stress can be obtained by means of the CUFSM proposed by Schafer [30]. The software CUFSM requires that the cross section of the analyzed member is uniform along the longitudinal direction. Obviously, the CFS built-up I-sectional members connected by the screws cannot meet this condition. Therefore, the CFS built-up I-sectional members cannot be directly calculated by using CUFSM. The simplified elastic buckling model needs to be proposed to predict the elastic critical buckling stress of built-up I-sectional columns under axial compression.

A single lipped C-section was used to determine the elastic local buckling stress of the CFS built-up I-sectional column by ignoring the restraint of screws on the elastic local buckling because the local buckling half-wavelength of a built-up column is usually smaller than the spacing of screws. On the contrary, the distortional buckling and overall buckling half-wavelengths of a built-up column are generally larger than the spacing of screws, and the torsion stiffness of the built-up member can be significantly improved by connecting two webs as a whole. Therefore, The elastic distortional buckling and overall buckling stress of the CFS built-up I-sectional columns can be determined by considering double C-sections as a whole I-section, as shown in Figure 22.

The ultimate strengths determined by using DSM design formulas (2)-(5) and approximate elastic buckling stress were compared with those obtained from tests as listed in Table 4. The comparison between the design curve of DSM and the load capacities obtained from the test and the FE parametric analyses is displayed in Figure 23. The mean value of test-to-prediction ultimate strength ratio $\left(P_{\mathrm{t}} / P_{\mathrm{D}}\right)$ is 0.744 with the corresponding coefficient of variation of 0.077 , as shown in Table 4 . The calculated ultimate strengths of built-up I-sections by using DSM are lower than that of the test and FEA results, as illustrated in Table 4 and Figure 23.

5.2. Modified Direct Strength Method. Since the interaction buckling strength of local and global buckling $\left(P_{\mathrm{nl}}\right)$ is always greater than or equal to global buckling strength $\left(P_{\mathrm{ne}}\right)$, the nominal ultimate strength of the CFS member is actually equal to the lower value of the interaction buckling strength between local and global $\left(P_{\mathrm{nl}}\right)$ and distortional buckling strength $\left(P_{\text {nd }}\right)$. Therefore, the interaction buckling strength curve between local and global and distortional buckling strength curve are modified in this section. The prediction formulas are as follows: 


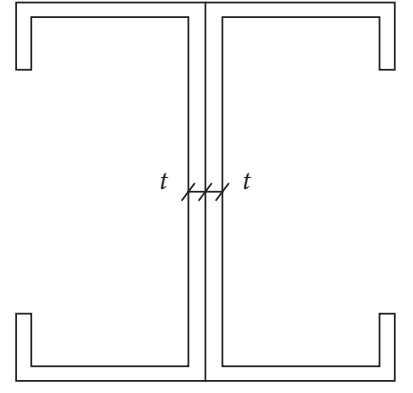

(a)

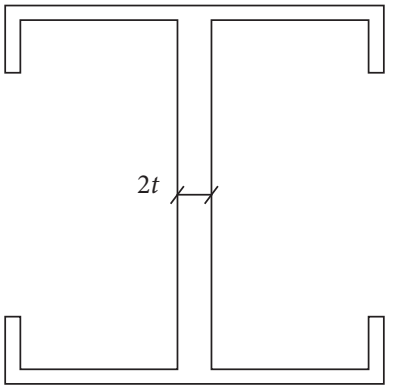

(b)

FIgURE 22: Cross section assumptions of the built-up I-section. (a) Double C-sections. (b) A whole I-section.

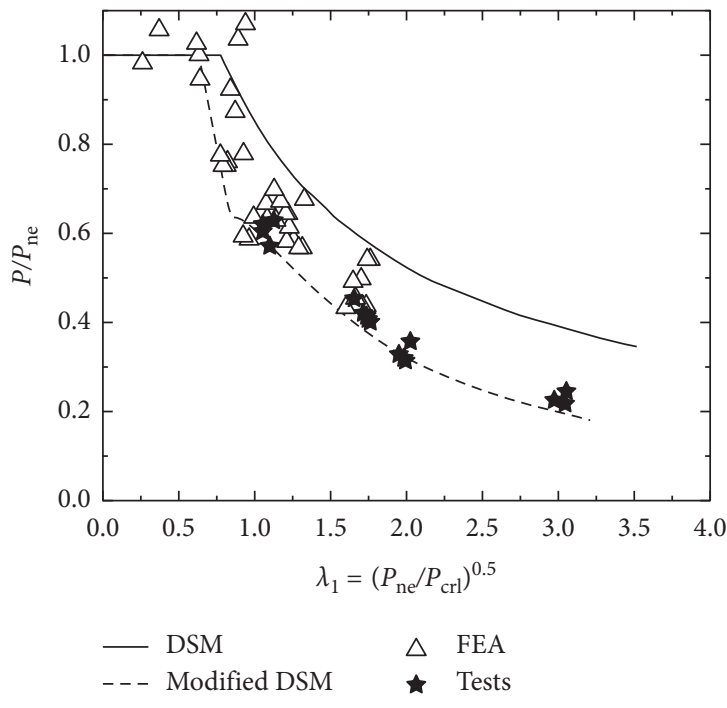

(a)

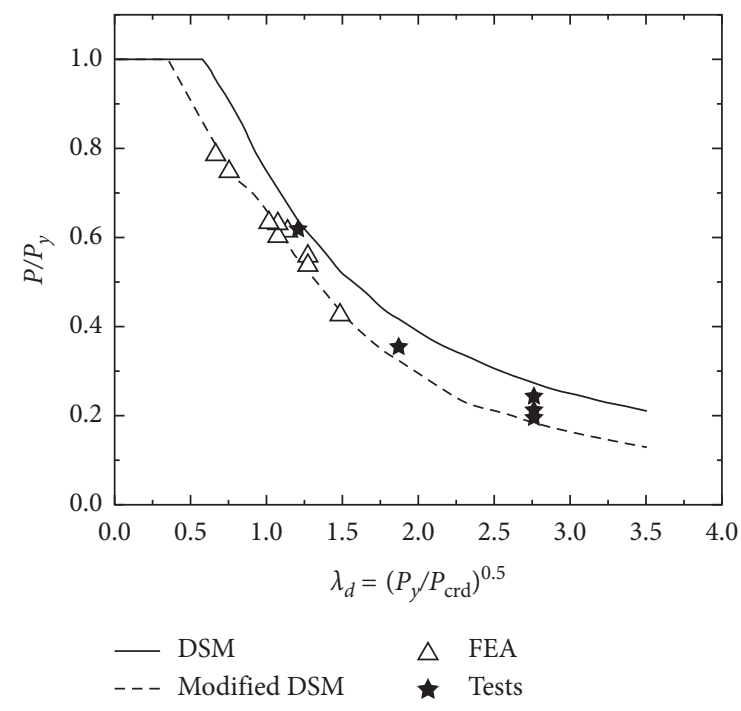

(b)

Figure 23: Comparison of the test results, FEA results with the DSM curves, and modified DSM curves. (a) Local buckling and global buckling interaction. (b) Distortional buckling.

$$
P_{M \mathrm{D}}=\min \left\{P_{m \mathrm{nl}}, P_{m \mathrm{nd}}\right\}
$$

$$
P_{m \mathrm{nl}}= \begin{cases}P_{\mathrm{ne}}, & \lambda_{l} \leq 0.636, \\ {\left[2.13-1.777\left(\frac{P_{\mathrm{crl}}}{P_{\mathrm{ne}}}\right)^{0.5}\right] P_{\mathrm{ne}},} & 0.636<\lambda_{l}<0.838, \\ {\left[1-0.39\left(\frac{P_{\mathrm{crl}}}{P_{\mathrm{ne}}}\right)^{0.7}\right]\left(\frac{P_{\mathrm{crl}}}{P_{\mathrm{ne}}}\right)^{0.7} P_{\mathrm{ne}},} & \lambda_{l} \geq 0.838,\end{cases}
$$

$$
P_{\mathrm{mnd}}= \begin{cases}P_{y}, & \lambda_{d} \leq 0.353, \\ \left(1.216-0.612\left(\frac{P_{\mathrm{crd}}}{P_{y}}\right)^{0.5}\right) P_{y}, & 0.353<\lambda_{d}<0.786, \\ {\left[1-0.34\left(\frac{P_{\mathrm{crd}}}{P_{y}}\right)^{0.8}\right]\left(\frac{P_{\mathrm{crd}}}{P_{y}}\right)^{0.8} P_{y},} & \lambda_{d} \geq 0.786 .\end{cases}
$$

The ultimate strength determined by using modified DSM design formulas (6)-(8) and the approximate elastic buckling stress were compared with the ultimate capacities obtained from tests as listed in Table 4. The comparison between the design curve of the modified DSM and the load capacities obtained from the test and the FE parametric analyses results are shown in Figure 23. The mean value of the test-to-modified prediction ultimate strength ratio $\left(P_{\mathrm{t}}\right)$ $\left.P_{\mathrm{MD}}\right)$ is 1.066 with the corresponding coefficient of variation of 0.06 , as shown in Table 4 . It indicated that the calculated ultimate strengths of built-up I-sections by using modified DSM are safe and accurate, and the modified DSM can be used to determine the ultimate strength of the CFS built-up I-sectional columns under axial compression.

\section{Conclusion}

The built-up I-section connected together with two lipped channel sections by screws through the webs is the most common built-up members used in CFS building. A total of 
56 CFS built-up I-sectional columns were tested. Based on the experimental investigation, numerical simulation, and theoretical analysis, some conclusions can be drawn as follows.

The interaction of local and distortional buckling and interaction of local, distortional, and overall buckling were observed in this test study. This interaction buckling had a significantly affect on the buckling behavior of CFS built-up I-sectional columns under axial compression. The built-up columns showed great composite action through the web screws. The spacing of screws and the end fastener group had a certain effect on ultimate strength of built-up columns, especially for columns failed with global buckling.

Numerical simulations for buckling modes and ultimate capacities of built-up I-sectional members were reliable and accurate.

The finite element parametric analysis indicated that the slenderness ratio had great influence on ultimate strength and stiffness for the built-up I-sectional members failed with local-distortional-overall buckling interaction and a few effect for the built-up I-sectional members failed with localdistortional buckling interaction. The spacing of screws and the end fastener group had a certain influence on ultimate strength of CFS built-up I-sectional columns when the builtup members had large spacing of screws.

The ultimate strength predicted using the proposed direct strength method in this paper can agree well with the test results and finite element results when the elastic distortional buckling and the elastic global buckling stress of the CFS built-up I-sectional columns are predicted by keeping the built-up cross section as a whole I-section, and a single C-section is used to determine the elastic local buckling stress. The comparison indicated that the proposed DSM design method is reliable for the common CFS built-up I-sectional columns in this paper. It needs to further verify for other CFS built-up I-sectional columns.

\section{Data Availability}

The data used to support the findings of this study are included within the article, and requests for data, 12 months after publication of this article, will be considered by the corresponding author.

\section{Conflicts of Interest}

The author declares that there are no conflicts of interest regarding the publication of this paper.

\section{Acknowledgments}

This research was funded by National Natural Science Foundation of China (Grant no. 51868049), Natural Science Foundation of Jiangxi Province in China (Grant no. 20181BAB206040), and Department of Education Science and Technology Projects of Jiangxi Province in China (Grant no. GJJ180932 and GJJ170983).

\section{References}

[1] JGJ227-2011, Technical Specification for Low-Rise ColdFormed Thin-Walled Steel Building, China Architecture \& Building Press, Beijing, China, 2011, in Chinese.

[2] AISI S100-2016, American Iron and Steel Institute. North American Specification for the Design of Cold-Formed Steel Structural Members, American Iron and Steel Institute, Washington, DC, USA, 2016.

[3] T. A. Stone and R. A. Laboube, "Behavior of cold-formed steel built-up I-sections," Thin-Walled Structures, vol. 43, no. 12, pp. 1805-1817, 2005.

[4] J. Whittle and C. Ramseyer, "Buckling capacities of axially loaded, cold-formed, built-up C-channels," Thin-Walled Structures, vol. 47, no. 2, pp. 190-201, 2009.

[5] K. Roy, T. C. H. Ting, H. H. Lau, and J. B. P. Lim, "Effect of thickness on the behaviour of axially loaded back-to-back cold-formed steel built-up channel sections-experimental and numerical investigation," Structures, vol. 16, pp. 327-346, 2018.

[6] F. C. David, T. Shahabeddin, Z. Xi et al., "Experiments on the global buckling and collapse of built-up cold-formed steel columns," Journal of Constructional Steel Research, vol. 144, pp. 65-80, 2018.

[7] F. C. David, T. Shahabeddin, Z. Xi et al., "Experimental study on the composite action in sheathed and bare built-up cold-formed steel columns," Thin-Walled Structures, vol. 127, pp. 290-305, 2018.

[8] T. Zhou, Y. Lu, W. Li, and H. Wu, "End condition effect on distortional buckling of cold-formed steel columns with arbitrary length," Thin-Walled Structures, vol. 117, pp. 282-293, 2017.

[9] Y. Lu, W. Li, T. Zhou, and H. Wu, "Novel local buckling formulae for cold-formed C-section columns considering end condition effect," Thin-Walled Structures, vol. 116, pp. 265276, 2017.

[10] M. Anbarasu and M. Venkatesan, "Behaviour of cold-formed steel built-up I-section columns composed of four U-profiles," Advances in Structural Engineering, vol. 22, no. 3, pp. 613-625, 2019.

[11] M. Anbarasu, "Numerical investigation on behaviour and design of cold-formed steel built-up column composed of lipped sigma channels," Advances in Structural Engineering, vol. 22, no. 8, pp. 1817-1829, 2019.

[12] S. Nie, T. Zhou, X. Zhou et al., "Experimental study and numerical analysis on double limb cold-formed steel built-up closed section columns under compression," Journal of Building Materials and Structures, vol. 38, no. 10, pp. 10-20, 2017, in Chinese.

[13] Y. Li, X. Yao, and Z. Shen, "Load-carrying capacity of $550 \mathrm{MPa}$ high-strength cold-formed thin-walled steel built-up box section columns," Building Structure, vol. 41, no. 6, pp. 36-41, 2011, in Chinese.

[14] Y. Li, Y. Li, S. Wang, and Z. Shen, "Ultimate load-carrying capacity of cold-formed thin-walled columns with built-up box and I section under axial compression," Thin-Walled Structures, vol. 79, pp. 202-217, 2014.

[15] I. Georgieva, L. Schueremans, and L. Pyl, "Composed columns from cold-formed steel Z-profiles: experiments and code-based predictions of the overall compression capacity," Engineering Structures, vol. 37, pp. 125-134, 2012.

[16] I. Georgieva, L. Schueremans, G. De Roeck, and L. Pyl, "Experimental investigation of the deformation of built-up members of cold-Formed steel profiles," Applied Mechanics and Materials, vol. 70, pp. 416-421, 2011. 
[17] I. Georgieva, L. Schueremans, L. Pyl, and L. Vandewalle, "Numerical study of built-up double-Z members in bending and compression," Thin-Walled Structures, vol. 60, pp. 85-97, 2012.

[18] I. Georgieva, L. Schueremans, L. Vandewalle, and L. Pyl, "Design of built-up cold-formed steel columns according to the direct strength method," Procedia Engineering, vol. 40, pp. 119-124, 2012.

[19] F. Liao, H. Wu, R. Wang, and T. Zhou, "Compression test and analysis of multi-limbs built-up cold-formed steel stub columns," Journal of Constructional Steel Research, vol. 128, pp. 405-415, 2017.

[20] X. Liu and T. Zhou, "Research on axial compression behavior of cold-formed triple-lambs built-up open T-section columns," Journal of Constructional Steel Research, vol. 134, pp. 102-113, 2017.

[21] S. Nie, T. Zhou, D. Yang et al., "Axial bearing capacity design method of cold-Formed steel quadruple-C built-up section members," Journal of Civil, Construction and Environmental Engineering, vol. 33, no. 4, pp. 20-28, 2011, in Chinese.

[22] M. Anbarasu and M. Adil Dar, "Improved design procedure for battened cold-formed steel built-up columns composed of lipped angles," Journal of Constructional Steel Research, vol. 164, Article ID 105781, 2020.

[23] M. Anbarasua and M. Adil Dar, "Axial capacity of CFS builtup columns comprising of lipped channels with spacers: nonlinear response and design," Engineering Structures, vol. 213, Article ID 110559, 2020.

[24] F. J. Meza, J. Becque, and I. Hajirasouliha, "Experimental study of the cross-sectional capacity of cold-formed steel built-up columns," Thin-Walled Structure, vol. 155, Article ID 106958, 2020.

[25] S. Nie, T. Zhou, M. R. Eathertonb et al., "Compressive behavior of built-up double-box columns consisting of four cold-formed steel channels," Engineering Structures, vol. 222, Article ID 111133, 2020.

[26] K. J. Rasmussen, M. Khezri, B. W. Schafer et al., "The mechanics of built-up cold-formed steel members," Thin-Walled Structure, vol. 154, Article ID 106756, 2020.

[27] GB/T 228.1-2010, Metallic Materials-Tensile Testing Part 1: Method of Test at Room Temperature, Standards Press of China, Beijing, China, 2011, in Chinese.

[28] X. Yao, Tests and Design Method on Distortional Buckling Behavior of Cold-Formed Thin-Walled Steel Built-Up I-Section Columns under Axial Compression, Nanchang Institute of Technology, Nanchang, China, 2020, in Chinese.

[29] ABAQUS Version 6.14-2, SIMULIA, Johnston, RI, USA, 2018.

[30] B. W. Schafer, CUFSM4.05-Finite Strip Buckling Analysis of Thin-Walled Members, Department of Civil Engineering, Johns Hopkins University, Baltimore, MD, USA, 2012. 